\title{
Design of zone-based bandwidth management scheme in IEEE 802.16 multi-hop relay networks
}

\author{
Yi-Ting Mai ${ }^{*}$ and Kuo-Yang Chen ${ }^{2}$
}

\begin{abstract}
IEEE 802.16 Wireless Network technology is a hot research issue in recent years. It provides wider coverage of radio and higher speed wireless access, and Quality-of-Service plays an important part in the standard. For mobile multihop wireless network, IEEE 802.16j/MR network not only can supply large area wireless deployment, but also can provide high quality network service to mobile users. Although Mobile QoS supporting has been extensively investigated, Mobile QoS supporting in the IEEE 802.16-MR network is relatively unexplored. In this article, the probability of a mobile user who visits a Relay Station (RS) is known beforehand. With the visiting probability at each RS and the system specified size of the range for bandwidth allocation, Base Station (BS) can calculate the required bandwidth to meet the mobile user's demand and allocate appropriate bandwidth for a mobile user roaming in the range of the bandwidth allocation. The range of bandwidth allocation for mobile users is called the Zone in this article, which includes the user's current RS and the nearby RSs. The proposed scheme is therefore called Zone-based bandwidth management scheme. The simulation results demonstrate that Zone-based bandwidth management scheme can reduce QoS degradation and bandwidth re-allocation overhead.
\end{abstract}

Keywords: 802.16, WiMAX, MR, Mobility, QoS

\section{Introduction}

With the popularity of wireless environments in recent years, multimedia applications such as the IPTV and MOD are more and more attractive to the mobile host $(\mathrm{MH})$ in the wireless networks like the IEEE $802.11[1,2]$ wireless LAN (WLAN) and the third generation (3G) [3,4] HSDPA (3.5 G) [5] cellular phone system. For large area and high bandwidth wireless transmission service, Broadband Wireless Access (BWA) technology is aiming to provide an easy, timesaving, and low cost method for deployment of next generation (beyond 4G) network infrastructure. IEEE 802.16 working group has launched a standardization process called Wireless Metropolitan Area Network (Wireless MANTM) for Broadband wireless access (BWA). BWA technology based on IEEE 802.16d (802.16-2004) [6] has been developed to achieve high speed mobile wireless network service to mobile users. Considering user mobility, IEEE 802.16e [7], 802.16-2009 [8], had also been

\footnotetext{
* Correspondence: wkb@mail.hit.edu.tw

${ }^{1}$ Department of Information and Networking Technology, Hsiuping Institute of Technology, Taiwan, Republic of China

Full list of author information is available at the end of the article
}

completed to support wireless access with high mobility. However, IEEE $802.16 \mathrm{e} / 802.16-2009$ only provides single hop wireless connectivity. So the latest version, IEEE 802.16j-2009 [9] was proposed for mobile multi-hop relay (MMR) networks. In an MMR network, Mobile Stations (MSs) are allowed to route through intermediate RSs to reach the BS, which differs from the single hop WiMAX topology. The new MMR network architecture imposes a demanding performance requirement on RSs. These relays will functionally serve as an aggregating point on behalf of the BS for traffic collection from and distribution to the multiple MSs associated with them. In the standard of IEEE 802.16j-2009, packet construction and delivery mechanism are inherited from IEEE 802.16/16e standard. The new multi-hop wireless network is called IEEE 802.16-MR in this article.

IEEE 802.16-MR enables fast network deployment in a large area at a lower cost than the traditional wired counterpart. Mobile users equipped with the IEEE 802.16 interface (WiMAX users, e.g., $\mathrm{MS}_{1}, \mathrm{MS}_{2}$ in Figure 1) can directly access the network while roaming in the area. IEEE 802.11 access point (Wi-Fi AP) connected to the RS is required for Wi-Fi users (e.g., $\mathrm{MH}_{1}, \mathrm{MH}_{2}$ in

\section{SpringerOpen ${ }^{\circ}$}

(C) 2011 Mai and Chen; licensee Springer. This is an Open Access article distributed under the terms of the Creative Commons Attribution License (http://creativecommons.org/licenses/by/2.0), which permits unrestricted use, distribution, and reproduction in any medium, provided the original work is properly cited. 


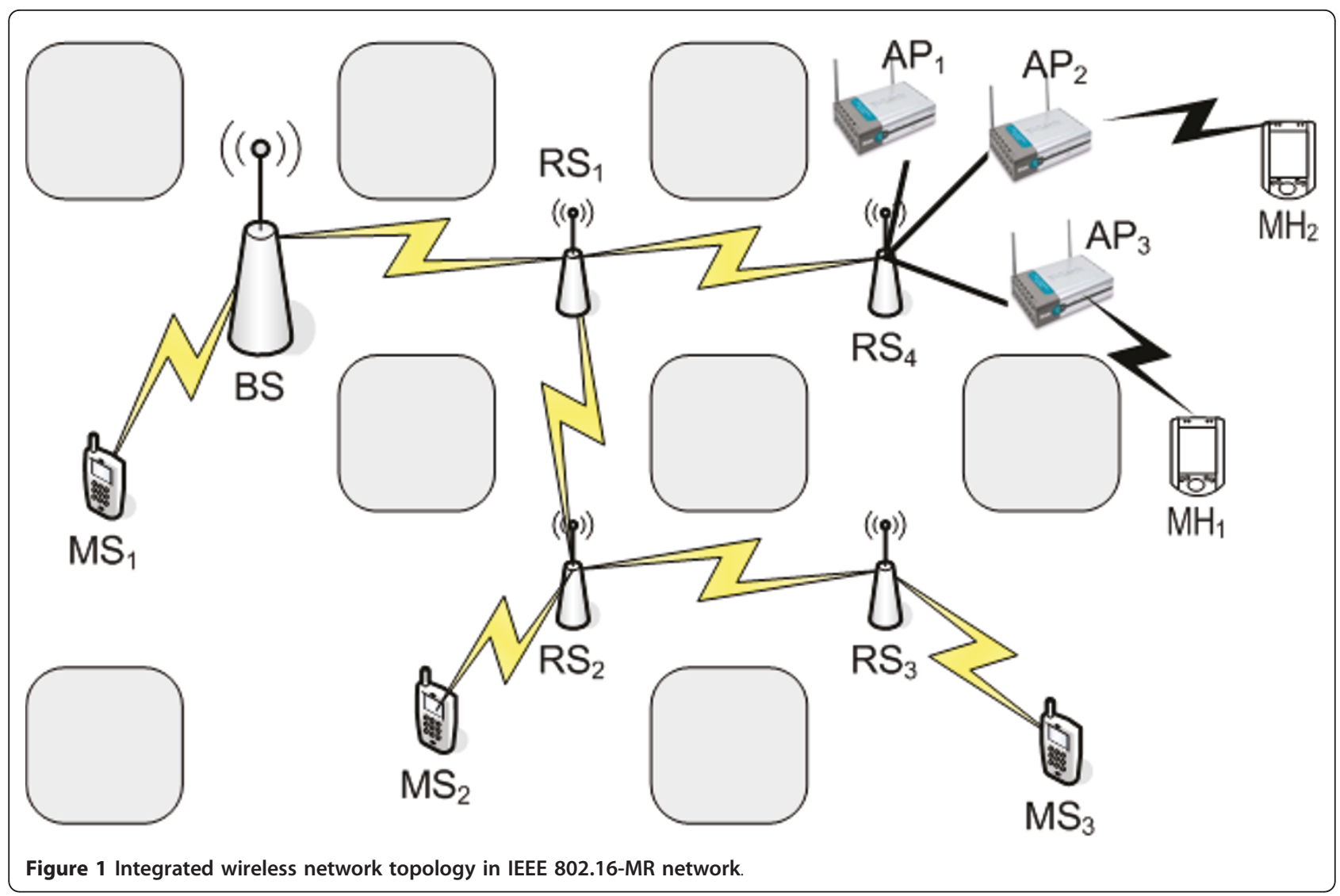

Figure 1) to gain access of the network. In either case of WiMAX or Wi-Fi users, an appropriate bandwidth allocation scheme in the IEEE 802.16-MR network is expected in order to guarantee QoS transmission for mobile users. There has been an increasing interest in QoS supporting for mobile users (also referred as Mobile QoS), which has been addressed in the literature for many years. However, the typical strategy for Mobile QoS is to reserve necessary bandwidth at neighboring nodes before the mobile user handoff to the new node, which inevitably results in low bandwidth utilization.

While all seems to agree that Mobile QoS is to reserve necessary bandwidth for next possible locations, opinions differ as to the different nature in network technology. Supporting of Mobile QoS in the IEEE 802.16MR network is worth a second thought. First of all, all RSs in the network share the same medium (channel), and the bandwidth requirement for a traffic flow depends on (more specifically, is proportional to) its path length (the number of RSs en route). Therefore, the bandwidth requirement of a mobile user at current $\mathrm{RS}$ is correlated with the bandwidth requirement at neighboring or nearby RSs. Secondly, the medium in the IEEE $802.16-\mathrm{MR}$ is managed by the BS in a centralized control manner, which provides the feasibility of more sophisticated bandwidth management in the network. The correlation of required bandwidth at nearby RSs leads to the idea of Zone-based bandwidth management proposed in this article. The zone of bandwidth allocation for a mobile user includes the user's current RS and the nearby RSs. The number of RSs in a zone is determined by the zone size, whose impact on different performance criteria has been investigated. Simulation study has shown the flexibility as well as the efficiency of the proposed scheme.

The remainder of the article is organized as follows. First, a survey of research works on the 802.16 QoS and mobile QoS are presented in 'Related works' section. The proposed Zone-based bandwidth management scheme in the 802.16-MR network is presented in 'Zone-based bandwidth management scheme' section. Simulation study for performance evaluation and comparison is presented in 'Performance evaluation' section. Finally, 'Conclusion' section concludes this article.

\section{Related works} IEEE 802.16 QoS

Recent QoS research has suggested that IEEE 802.16 wireless network may indeed facilitate processes beneficial to achieve mobile multimedia application. Basic 
QoS service types have been proposed in the IEEE 802.16 standard [6,7], such as five service types, Unsolicited Grant Service (UGS), extend real-time Polling Service (ertPS), real-time Polling Service (rtPS), non-realtime Polling Service (nrtPS), and Best Effort (BE). A specific scheduling algorithm is not described in the IEEE 802.16 standard, so some mechanisms of QoS support such as admission control and bandwidth allocation in IEEE 802.16 were extensively researched in the literature. Based on the connection-oriented concept, the admission control scheme $[10,11]$ must be properly designed to decide whether a new request of traffic flow can be granted or not. The new request is granted only when the bandwidth requirement of the request can be satisfied and none of the quality of the existing traffic flows is violated. On the other hand, some research articles $[12,13]$ proposed scheduling mechanism for bandwidth allocation in IEEE 802.16. The common idea of these scheduling mechanisms is to dynamically allocate time slots according to the service type of the traffic flows and to achieve higher network utilization. To integrate IP layer scheduling (L3) and IEEE 802.16 scheduling (L2), Chen et al. $[14,15]$ proposed the idea of multilayer QoS scheduling support by assigning different scheduling algorithms in L3 and L2 for different combinations of L3 and L2 service types.

The Mesh mode in IEEE 802.16 network provides the Subscriber Stations (SSs) not only for connecting to BS but also other SSs directly. Since the SSs could connect to each other and BS, the network management is different in 802.16 PMP mode. Since the IEEE 802.16 standard is a Layer 1 and Layer 2 protocol, it does not specify how the traffic will be routed in the mesh topology. In Centralized scheduling research works [16-19], different scheduling and routing mechanisms were proposed to improve the performance by lowering the interference of routes and reducing the congestion near the hotspot of the BS. However, longer path introduces more link consumption, which further causes a significant decrease in network utilization. For designing QoS mechanisms, most of the Centralized-based research works $[20,21]$ focused on the construction of the routing tree based on different QoS types. Distributed scheduling provides better routing path without always requiring the traffic going via the BS. In Distributed scheduling, each node competes for channel access using a pseudorandom election algorithm based on the scheduling information of the two hop neighbors. However, the complicated behavior of Distributed scheduling makes it difficult to provide precise bandwidth allocation, which also makes it inappropriate in QoS support [22].

Since IEEE 802.16-MR network is multi-hop topology, network utilization, route selection, resource allocation and handoff issue should be discussed. To improve the system utilization, some research works [23-25] focus on medium access control (MAC) and radio resource management problems in IEEE 802.16j networks. References $[26,27]$ addressed the path selection, link scheduling and routing problem in IEEE 802.16j networks considering metrics such as number of hop count and maximum E2E throughput. Considering QoS supporting and bandwidth allocation, bandwidth allocation schemes were proposed for 802.16-MR networks in order to satisfy traffic demand from different flow requests and guarantee QoS demands of different applications [28,29].

In order to achieve QoS support in IEEE 802.16 network, both 802.16 layer and upper layer QoS should be considered. Cross layer QoS frameworks for IEEE PMP [30] and Mesh [31] were proposed, respectively, in our previous work. Higher throughput, lower access delay and less signaling overhead can be achieved in the frameworks. QoS supporting for mobile users is not addressed in most of the previous works on IEEE 802.16 QoS, let alone Mobile QoS supporting in the IEEE 802.16-MR network. Traditional networks generally require the use of RSVP to reserve bandwidth for users. Some research articles [32,33] applied the RSVP concept for E2E QoS reservation in IEEE 802.16 Mesh network, but they cannot support frequent $\mathrm{MH}$ handoff. Mobile RSVP (MRSVP) [34] is an extension of RSVP that distinguishes between two kinds of reservations: the active and passive reservations. Hierarchical Mobile RSVP (HMRSVP) [35] integrates RSVP with Mobile IP regional registration protocol [36], in which the $R S V P$ session between the $\mathrm{MH}$ and the $\mathrm{CN}$ is split into 2-tier group.

\section{Bandwidth reservation with Mobile QoS}

Traditional RSVP based mechanisms for Mobile QoS are Internet wide and operate above the IP layer. It is extremely challenging to allocate bandwidth for mobile users since QoS must be achieved over the E2E path in the presence of handoff. Furthermore, the IEEE.802.16MR network is operating under the IP layer, which classifies the handoff within the IEEE 802.16-MR network as Micro-Mobility. Resources management in IEEE 802.16-MR is centrally controlled by the BS. As illustrated in Figure 2, after MH handoff, the important thing to consider is whether the resources reserved for the $\mathrm{MH}$ is enough. In the case of the same hop count before and after handoff, the BS only needs to reassign the Mini-slots used by the RSs on the old path to the RSs on the new path without triggering bandwidth reallocation. Nevertheless in IEEE 802.16-MR, the reserved bandwidth must be enough to meet the requirements of the hop count between the BS and the current RS which the $\mathrm{MH}$ is connecting to. Considering the 


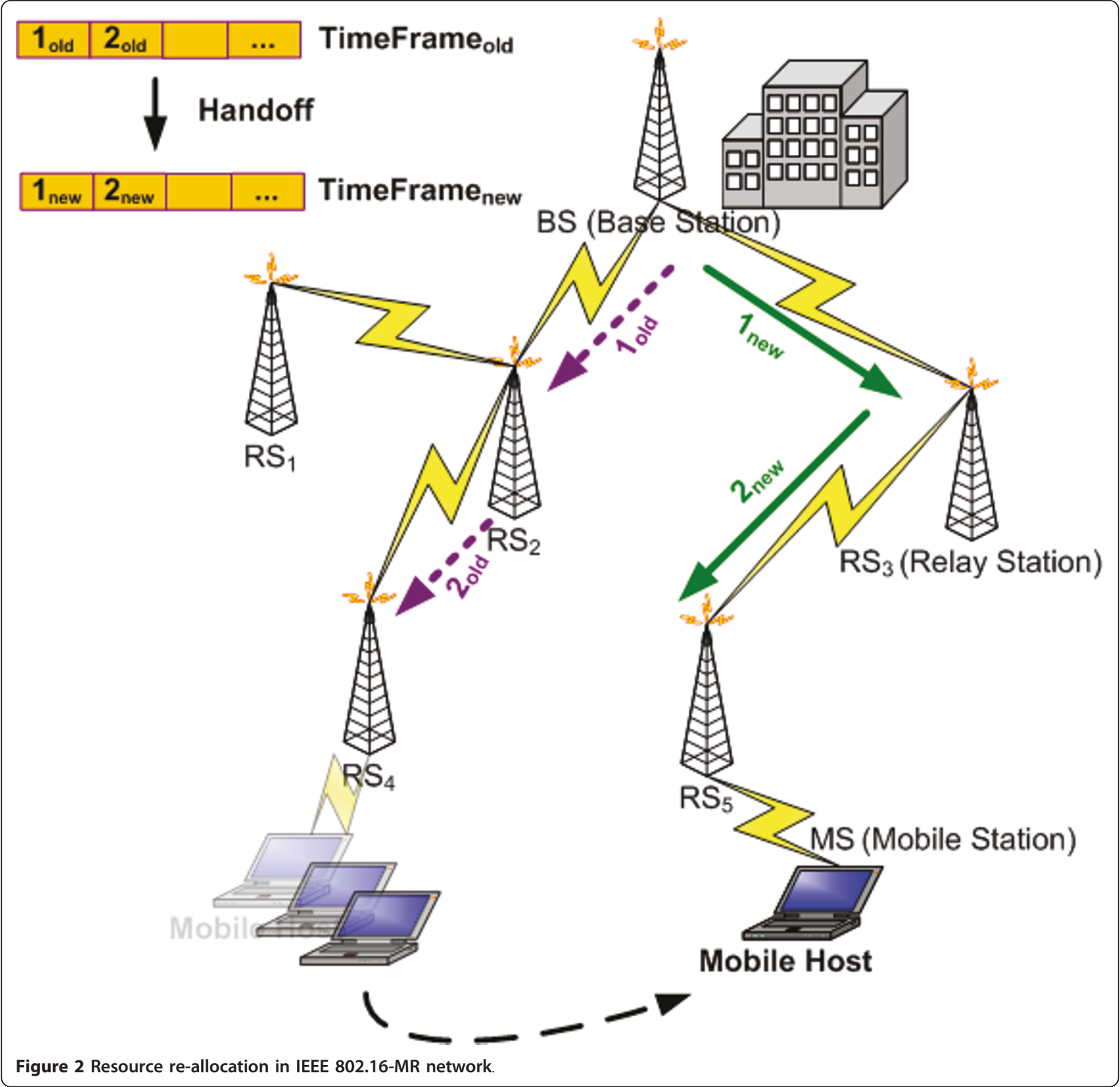

mobility issue, the scope of operations in traditional network is generally divided into two parts of Macro-Mobility and Micro-Mobility. As far as IEEE 802.16-MR network is concerned, we usually focus on the part of Micro-Mobility inside the IEEE 802.16-MR network domain. In general case, the BS has to ensure there is enough bandwidth for handoff, which leads to the idea of Zone-based bandwidth management in this article.

\section{Zone-based bandwidth management scheme} Basic idea and notations

The motivation of Zone-based bandwidth management is to reserve appropriate amount of bandwidth used for a mobile user at all RSs within the zone such that bandwidth re-allocation is not necessary for handoffs of the user among the RSs of the same zone as displayed in Figure 3. The size of a zone is defined to be the hop count of the most distant RS from the initial (center) RS. For more general purpose to cover different network sizes, a system parameter $L$, whose value is in between 0 and 1 , is defined for zone size in the article. Assuming the size of the IEEE 802.16-MR network in hop count is $H C_{M A X}$, zone size $L$ means the hop count of the most distant RS from the initial RS $\left(R S_{\text {initial }}\right)$ is $\left\lceil L^{*} H C_{M A X}\right\rceil$ as illustrated in Figure 4. Therefore, the zone only includes the initial RS for $L=0$, and all RSs in the network for $L$ 


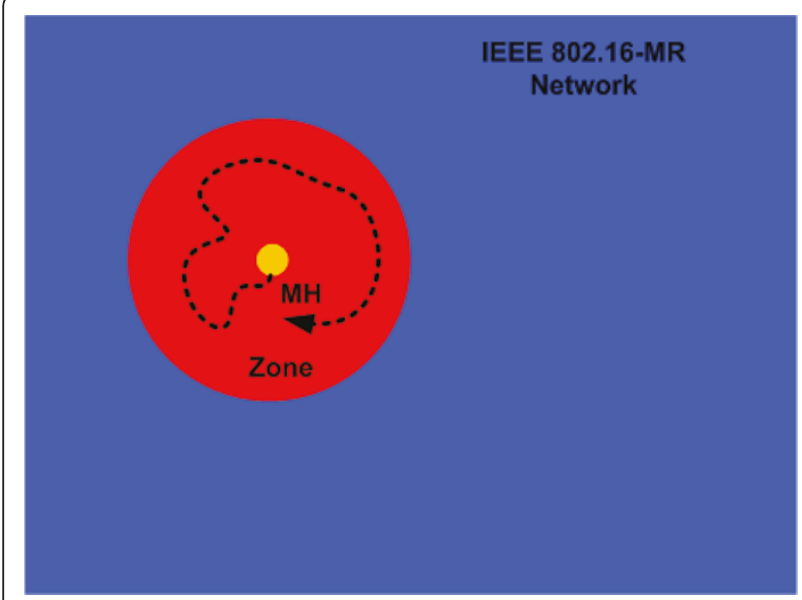

Figure 3 Zone area for MH movement.

$=1$. Following assumptions are made for better understanding the proposed scheme.

(1) All RSs in the network share the same medium without spatial reuse in medium access, i.e., two or more RSs cannot access the medium at the same time.

(2) BS is fully in charge of medium access control and is responsible for bandwidth allocation by using fields like $U L-M A P$ and $D L-M A P$ in the control subframe. Details of the signaling procedure as well as the exchange of control messages are not presented in the article.

(3) Although the proposed scheme can be applied to other types of topology, a chessboard topology as displayed in Figure 4 is used for modeling the network. BS is located at the upper left corner. The correspondent node $(\mathrm{CN})$ for the mobile user is located outside the network. The proposed scheme only considers bandwidth allocation within the IEEE 802.16-MR network.

(4) The visiting probability of the mobile user at each RS is assumed to be obtainable either by user profile data or network modeling techniques. The visiting probability of the mobile user at $\mathrm{RS} R S_{i, j}$ is denoted by $P_{\mathrm{RS}_{i, j}}$.

(5) The applications adopting the proposed scheme are assumed to be adaptable to bandwidth adjustment. The satisfaction rate for the required bandwidth, denoted by $S$, is defined as the ratio of the allocated bandwidth over the required value (i.e., Satisfaction $\left.=\frac{\text { Allocated Bandwidth }}{\text { Required Bandwidth }}\right)$. The mobile user provides the flow rate (denoted by $B W$ ) as well as the threshold of the satisfaction rate (denoted by $\left.S \_T H\right)$ for bandwidth allocation.
Notations used in the paper are summarized in Table 1.

\section{Admission control and bandwidth allocation}

Given the flow rate $B W$, the satisfaction threshold $S_{-} T H$, the zone size $L$, and the initial location of the mobile user $\mathrm{RS}_{\text {initial, }}$ we are showing the calculation of the allocated bandwidth. First of all, all RSs in the zone must be identified according to the value $L$ as follows.

$\mathrm{RS}_{i, j} \in$ Zone $_{\mathrm{RS}_{\text {initial }}, L}$

as long as the hop count between $\mathrm{RS}_{i, j}$ and $\mathrm{RS}_{\text {initial }} \leq\left\lceil\mathrm{HC}_{\mathrm{MAX}} * L\right\rceil$

Secondly, by normalization of the visiting probability at all RSs in the network, the visiting probability for each RS in the zone (denoted by $P_{\mathrm{RS}_{i, j}}^{Z \text { one }}$ ) can be obtained as follows. $P_{\mathrm{RS}}^{\mathrm{Zone}, j}=\frac{P_{\mathrm{RS}_{i, j}}}{\sum_{\forall \mathrm{RS} \text { in the Zone }} P_{\mathrm{RS}}}$

$P_{\mathrm{RS}_{i, j}}^{Z \text { one }}$ is the visiting probability of the mobile user at $\mathrm{RS}_{i, j}$ in the case of the user not moving outsize of the zone. If we assume the bandwidth allocated in the zone is $N^{*} B W$, the satisfaction rate $S$ for the allocation can be calculated as follows.

$$
S=\sum_{\forall \mathrm{RS} \text { in the Zone }}\left[\operatorname{Min}\left(1, \frac{N * \mathrm{BW}}{\mathrm{HC}_{\mathrm{RS}_{i, j}} * \mathrm{BW}}\right)\right] * P_{\mathrm{RS}_{i, j}}^{\mathrm{Zone}},
$$

where $\mathrm{HC}_{\mathrm{RS}_{i, j}}$ is the hop count between BS and $\mathrm{RS}_{i, j}$. Note that the satisfaction rate at each RS (calculated by $N * \mathrm{BW}$

$\left.\frac{\mathrm{N} * \mathrm{BW}}{\mathrm{HC}_{\mathrm{RS}} \text { i,j } \mathrm{BW}}\right)$ should be no larger than 1 . This is why the Min operator is placed in the above equation.

Finally, the allocated bandwidth is determined by the minimum value of $N$ which makes the value of $S$ in (Equation 1) larger than (or equal to) the threshold of the satisfaction rate $S_{-} T H$.

For example, given the following parameters, $S_{-} T H=$ 0.8 , Zone size $=3, \mathrm{RS}_{\text {initial }}=\mathrm{RS}_{5,5}$, the hop count of each RS in the zone as displayed in Figure 5, and the same visiting probability for all RSs, the value of satisfaction rate $S \approx 0.784$ for $N=8$ and $S \approx 0.828$ for $N=9$ according to the calculation of Equation 1. Bandwidth allocation for the zone of the case should be $9 * \mathrm{BW}$ to make value of $S$ greater than $S_{-} T H$.

Admission control for the new mobile user is simply by checking if current available bandwidth is enough for the calculated value of bandwidth allocation.

We would like to find the minimum $N$ which satisfies the equation above and makes $S^{*}$ larger than or equal to the user parameter $S$, where $\mathrm{HC}_{S S i, j}$ is the hop count length from RS to the $\mathrm{CN}$. The $N$ obtained represents that when we reserve bandwidth of $N * \mathrm{BW}$, the expected value of satisfaction within Zone would be larger than or equal to user parameter $S_{-} T H$. Thus, 


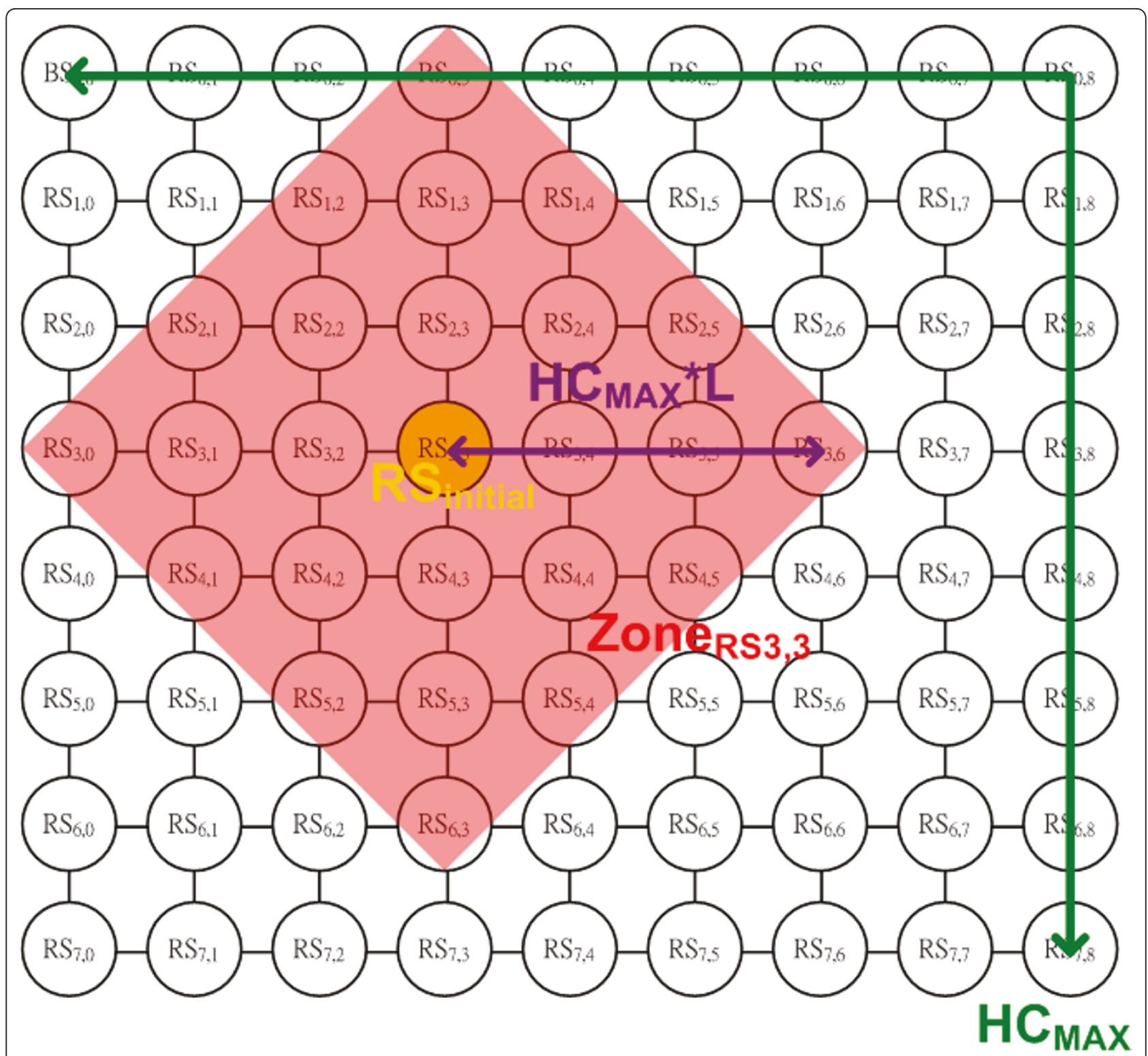

Figure 4 Zone coverage area of parameter $L$.

Table 1 Summary of notations.

\begin{tabular}{|c|c|c|}
\hline Notation & Description & Type \\
\hline L & Zone size & System parameters \\
\hline$S$ & Satisfaction rate for required bandwidth & \\
\hline$\overline{H C_{\text {MAX }}}$ & Hop count of $802.16-M R$ & \\
\hline$P_{\mathrm{RS}_{i, j}}$ & Visiting probability at $\mathrm{RS}_{i, j}$ & \\
\hline$P_{\mathrm{RS}_{i, j}}^{\text {Zone }}$ & Normalized visiting probability at $\mathrm{RS}_{i, j}$ within the zone & \\
\hline $\mathrm{HC}_{\mathrm{RS}_{i, j}}$ & Hop count between BS and $R S i, j$ & \\
\hline $\bar{S}$ STH & Threshold of the satisfaction rate & User parameters \\
\hline BW & Flow data rate & \\
\hline$\overline{R \text { initial }}$ & The initial RS of the zone for bandwidth allocation & \\
\hline
\end{tabular}




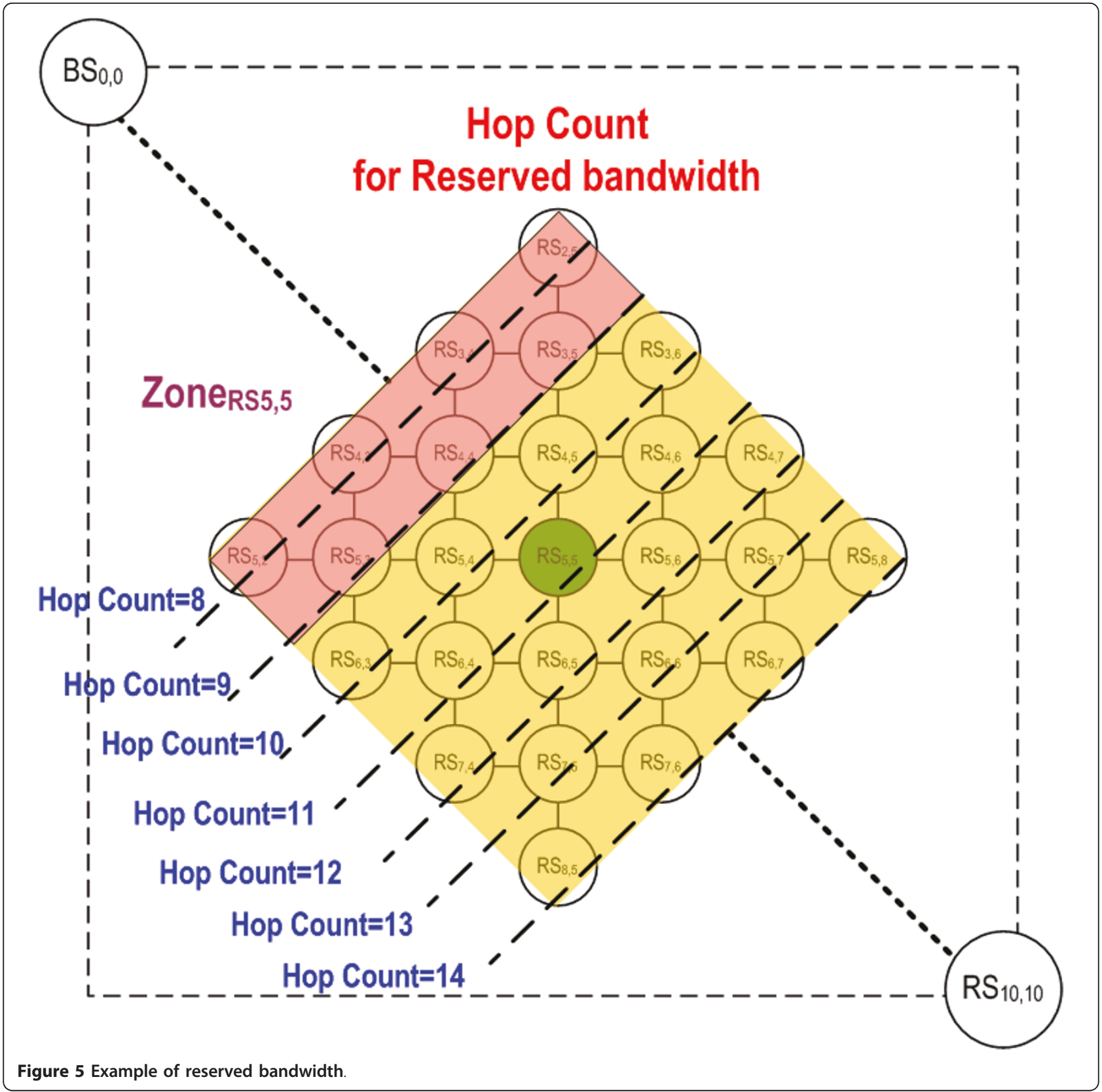

$N * \mathrm{BW}$ is the bandwidth we reserve for MHs. The admission control is determined by:

$$
\left\{\begin{array}{c}
\mathrm{BW}_{\text {remain }} \geq N * \mathrm{BW} \text {, accepted } \\
\mathrm{BW}_{\text {remain }}<N * \mathrm{BW} \text {, rejected }
\end{array}\right.
$$

\section{$\mathrm{BW}_{\text {remain }}$ :system remaining resource}

From Equation 2, when $\mathrm{BW}_{\text {remain }} \geq N$ * $\mathrm{BW}$, where $B W_{\text {remain }}$ represents the available bandwidth in the system, users are allowed to enter the system. Otherwise, they have to wait for the next time of BS bandwidth allocation.
Intra-zone handoff and inter-zone handoff

Moreover, by introduction the idea of zone, two types of handoff between RSs are defined, intra-zone handoff and inter-zone handoff as illustrated in Figure 6. Bandwidth re-allocation is only triggered by inter zone handoffs, and the RS triggering bandwidth re-allocation becomes the initial RS of the new zone.

For example, when a $\mathrm{MH}$ is moving toward the boundary of its zone as displayed in Figure 6. If the $\mathrm{MH}$ keeps on moving, it may be out of the zone. Therefore, it is necessary to determine immediately if the bandwidth reserved for this MH needs to be adjusted. The 


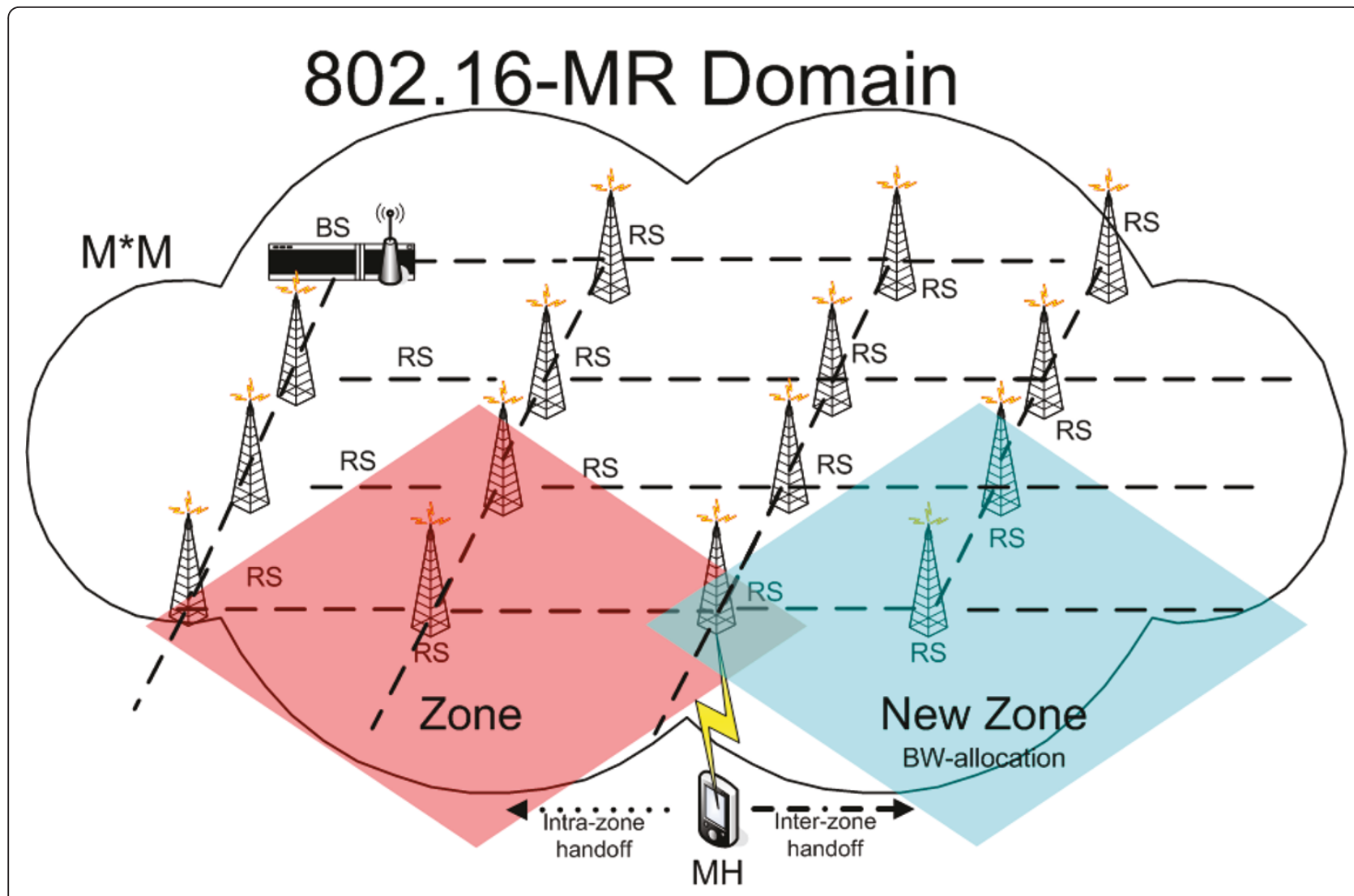

Figure 6 Intra-zone and Inter-zone handoff.

$\mathrm{RS}$ of the current location is considered as the new $\mathrm{RS}_{\mathrm{i}-}$ nitial to calculate again reserved bandwidth for this $\mathrm{MH}$. The new Zone will become the area with the current location being the center as shown in the figure. In summary, there are three possible situations:

\section{Case 1 (Reserved bandwidth of new location < original} reserved bandwidth)

When MH new position has a smaller hop count than original node's hop count, it can return some bandwidth. We calculate the system remaining resource according to the difference of $\mathrm{MH}$ original hop count and $\mathrm{MH}$ new hop count. $\mathrm{BW}_{\text {remain }}=\mathrm{BW}_{\text {remain }}+\mathrm{BW}$ * $N_{\text {diff }}\left(N_{\text {diff }}=N_{\text {old }}-N_{\text {new }}\right)$.

Case 2 (Reserved bandwidth of new location = original reserved bandwidth)

When MH new location has the same hop count as original MH's hop count, the system remaining resource do not need to modify.

original reserved bandwidth)", 1,0,2,0,0pc,0pc,0pc,0pc>Case 3 (Reserved bandwidth of new location > original reserved bandwidth)

When new location of $\mathrm{MH}$ has a larger hop count than original MH's hop count, it needs to request more bandwidth. So we should check the state of remaining resource. If $\mathrm{BW}_{\text {remain }}>\mathrm{BW} * N_{\text {diff }}\left(N_{\text {diff }}=N_{\text {new }}-N_{\text {old }}\right)$, we still have enough bandwidth for new request, $B W_{\text {re- }}$ main $=\mathrm{BW}_{\text {remain }}-B W^{*} N_{d s f}$. However, if the remaining bandwidth resource is not enough (i.e., $\mathrm{BW}_{\text {remain }}<\mathrm{BW}$ * $N_{\text {diff }}$ ), the $\mathrm{MH}$ can request BS to allocate more bandwidth in the next round.

\section{Performance evaluation}

\section{Simulation topology analysis}

In IEEE 802.16-MR network, the network topology might have different influences on network efficiency. This is because in fact, the higher hop count of a MH's current location RS, the more RSs are required for data transmission, and, for a multi-hop wireless network system, the more bandwidth is required. Thus, we try to analyze two popular types of topologies, one is chessboard topology and the other one is tree topology.

\section{Tree topology}

Figure 7 shows a tree topology with height $y($ root $=0)$ and degree $x$ in 802.16 -MR network. The MHs in the figure cannot only visit to their parent nodes and child nodes, but also their sibling nodes. This means when a $\mathrm{MH}$ moves in the topology, it can move to parent nodes, child nodes, and sibling nodes with equal 


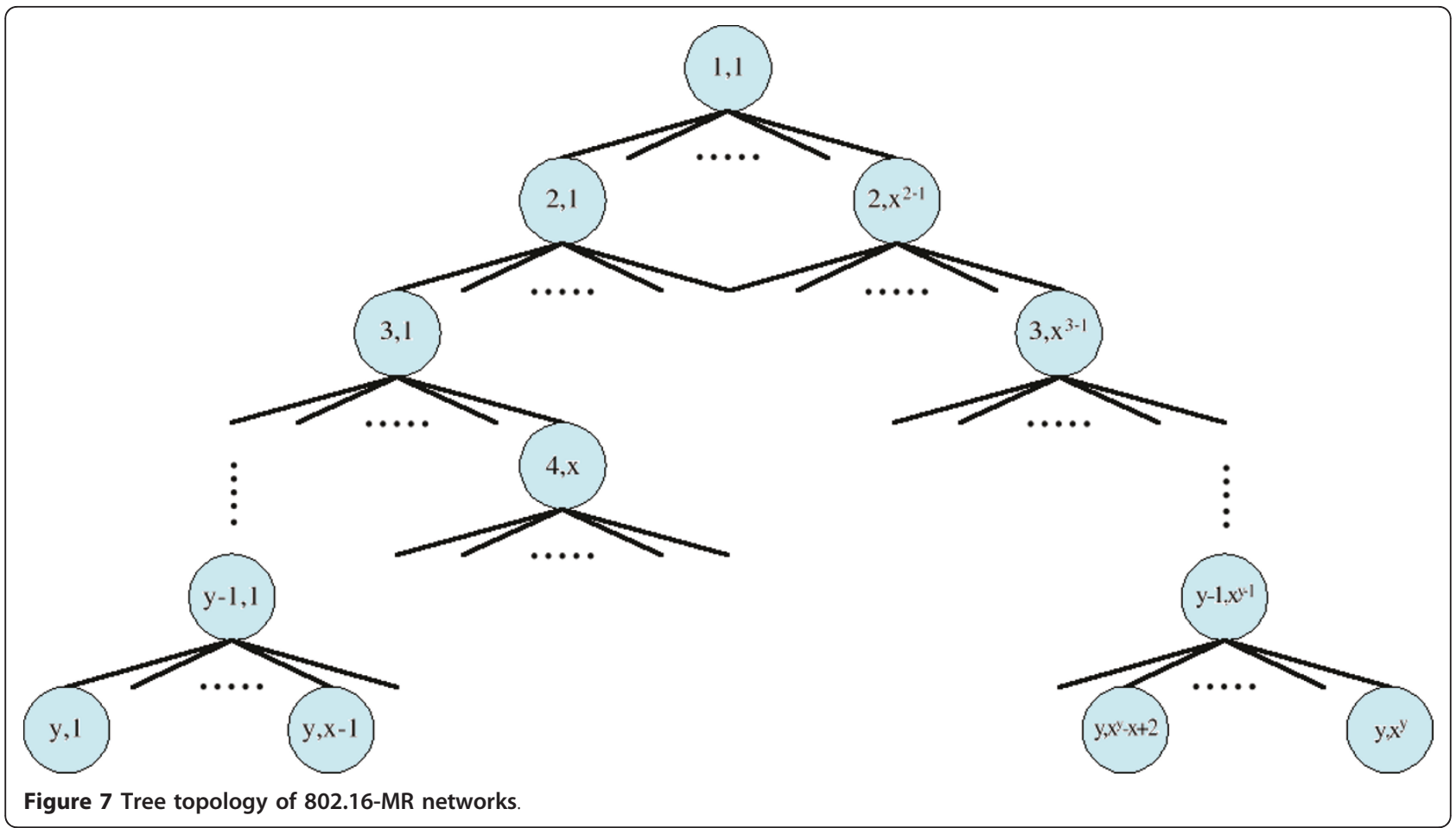

probability. Let $P_{\mathrm{MH}}(i, j)$ denotes the probability that the $\mathrm{MH}$ is at location $(i, j)$, and $p$ denotes the probability that the MH leaves its current network. According to the balance theorem in the queueing theory, we can write down the following equation.

$$
\begin{aligned}
& P_{\mathrm{MH}}(1,1)=P_{\mathrm{MH}}(1,1) \times(1-p)+P_{\mathrm{MH}}(2,1) \times \frac{p}{x+2} \\
& \quad+P_{\mathrm{MH}}(2,2) \times \frac{p}{x+3}+\ldots+P_{\mathrm{MH}}\left(2, x^{2-1}\right) \times \frac{p}{x+2} \\
& \quad=P_{\mathrm{MH}}(1,1) \times(1-p)+2 \times P_{\mathrm{MH}}(2,1) \times \frac{p}{x+2}+(x-2) P_{\mathrm{MH}}(2,2) \times \frac{p}{x+3} \\
& \text { and } \sum_{i=1}^{\gamma+1} \sum_{j=1}^{x^{\prime}} P_{\mathrm{MH}}(i, j)=1 .
\end{aligned}
$$

Since different locations of the tree node result in different transition patterns, we classify the tree nodes into five categories $(a \sim e)$ for analysis as illustrated in Figure 8:

(1) There is one root node, let $P_{\mathrm{MH}}(a)$ denote the probability that the $\mathrm{MH}$ is at root node.

(2) From the second level of the tree, let $P_{\mathrm{MH}}(b)$ denote the probability that the $\mathrm{MH}$ is at the left or right corner of subtree root. There are $(y-1) * 2$ tree nodes in this category.

(3) For the leaf level, let $P_{\mathrm{MH}}(c)$ denote the probability that the $\mathrm{MH}$ is at the left or right corner of leaf nodes. There are two tree nodes in this category.
(4) For leaf level, let $P_{\mathrm{MH}}(d)$ denote the probability that the $\mathrm{MH}$ is at the remaining leaf nodes. There are $x^{y}-2$ tree nodes in this category.

(5) Finally, the probability that the $\mathrm{MH}$ is at remaining tree nodes are called $P_{\mathrm{MH}}(e)$. There are $\left(x^{y}-2 x y\right.$ $+x+2 y-2) /(x-1)$ tree nodes in this category.

The Markov Chain for the tree topology is shown in Figure 9 , in which the number of neighbors for a tree mode in category $a$ is $x, x+2$ for category $b, 2$ for category $c, 3$ for category $d$, and $x+3$ for category $e$. According to the balance theorem, the relationship of tree nodes in each category is given in the following:

\section{Case a}

$$
P_{\mathrm{MH}}(a)=P_{\mathrm{MH}}(a) \times(1-p)+\frac{2 P_{\mathrm{MH}}(b) \times p}{x+2}+\frac{(x-2) \times P_{\mathrm{MH}}(e) \times p}{x+3}
$$

\section{Case $b$}

$$
\begin{aligned}
& P_{\mathrm{MH}}(b)=P_{\mathrm{MH}}(b) \times(1-p)+\frac{P_{\mathrm{MH}}(a) \times p}{x}+\frac{P_{\mathrm{MH}}(b) \times p}{x+2}+\frac{x \times P_{\mathrm{MH}}(e) \times p}{x+3} \\
& P_{\mathrm{MH}}(b)=P_{\mathrm{MH}}(b) \times(1-p)+\frac{P_{\mathrm{MH}}(a) \times p}{x}+\frac{(x+1) \times P_{\mathrm{MH}}(e) \times p}{x+3} \\
& P_{\mathrm{MH}}(b)=P_{\mathrm{MH}}(b) \times(1-p)+\frac{2 P_{\mathrm{MH}}(b) \times p}{x+2}+\frac{x \times P_{\mathrm{MH}}(e) \times p}{x+3} \\
& P_{\mathrm{MH}}(b)=P_{\mathrm{MH}}(b) \times(1-p)+\frac{P_{\mathrm{MH}}(b) \times p}{x+2}+\frac{P_{\mathrm{MH}}(c) \times p}{2} \\
& +\frac{(x-1) \times P_{\mathrm{MH}}(d) \times p}{3}+\frac{P_{\mathrm{MH}}(e) \times p}{x+3}
\end{aligned}
$$




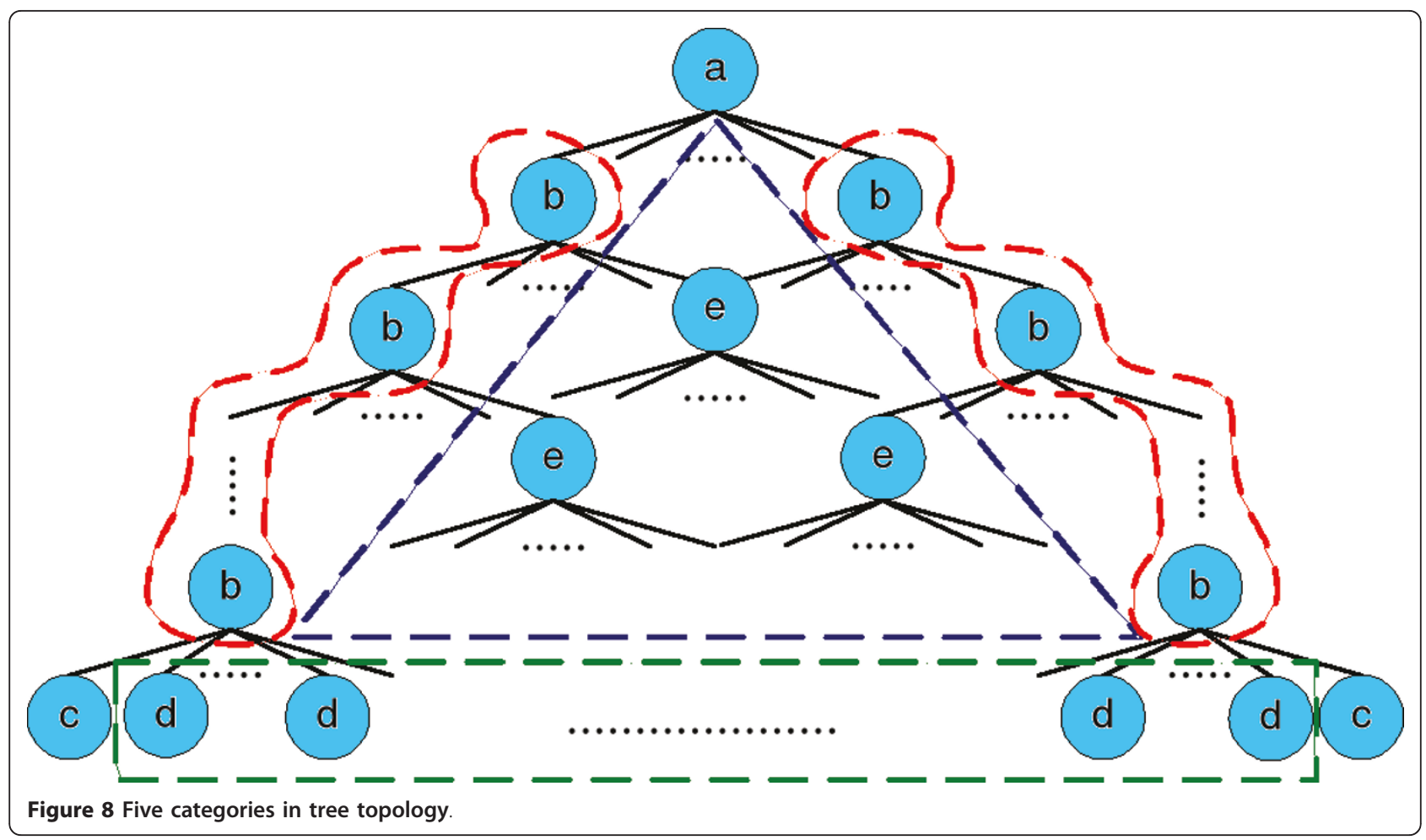

\section{Case c}

$$
P_{\mathrm{MH}}(c)=P_{\mathrm{MH}}(c) \times(1-p)+\frac{P_{\mathrm{MH}}(b) \times p}{x+2}+\frac{P_{\mathrm{MH}}(d) \times p}{3}
$$

\section{Case d}

$$
\begin{aligned}
& P_{\mathrm{MH}}(d)=P_{\mathrm{MH}}(d) \times(1-p)+\frac{P_{\mathrm{MH}}(b) \times p}{x+2}+\frac{P_{\mathrm{MH}}(c) \times p}{2}+\frac{P_{\mathrm{MH}}(d) \times p}{3} \\
& P_{\mathrm{MH}}(d)=P_{\mathrm{MH}}(d) \times(1-p)+\frac{P_{\mathrm{MH}}(b) \times p}{x+2}+\frac{2 P_{\mathrm{MH}}(d) \times p}{3} \\
& P_{\mathrm{MH}}(d)=P_{\mathrm{MH}}(d) \times(1-p)+\frac{2 P_{\mathrm{MH}}(d) \times p}{3}+\frac{P_{\mathrm{MH}}(e) \times p}{x+3}
\end{aligned}
$$

\section{Case e}

$$
\begin{aligned}
& P_{\mathrm{MH}}(e)=P_{\mathrm{MH}}(e) \times(1-p)+\frac{P_{\mathrm{MH}}(a) \times p}{x}+\frac{P_{\mathrm{MH}}(b) \times p}{x+2}+\frac{(x+1) \times P_{\mathrm{MH}}(e) \times p}{x+3} \\
& P_{\mathrm{MH}}(e)=P_{\mathrm{MH}}(e) \times(1-p)+\frac{(x+3) \times P_{\mathrm{MH}}(e) \times p}{x+3} \\
& P_{\mathrm{MH}}(e)=P_{\mathrm{MH}}(e) \times(1-p)+\frac{2 P_{\mathrm{MH}}(b) \times p}{x+2}+\frac{(x+1) \times P_{\mathrm{MH}}(e) \times p}{x+3} \\
& P_{\mathrm{MH}}(e)=P_{\mathrm{MH}}(e) \times(1-p)+\frac{2 P_{\mathrm{MH}}(b) \times p}{x+2}+\frac{x \times P_{\mathrm{MH}}(d) \times p}{3}+\frac{P_{\mathrm{MH}}(e) \times p}{x+3} \\
& P_{\mathrm{MH}}(e)=P_{\mathrm{MH}}(e) \times(1-p)+\frac{P_{\mathrm{MH}}(b) \times p}{x+2}+\frac{x \times P_{\mathrm{MH}}(d) \times p}{3}+\frac{2 P_{\mathrm{MH}}(e) \times p}{x+3}
\end{aligned}
$$

Based on the above five case equations, the relation of $P_{\mathrm{MH}}(a), P_{\mathrm{MH}}(b), P_{\mathrm{MH}}(c), P_{\mathrm{MH}}(d)$ with $P_{\mathrm{MH}}(e)$ can be shown as follows:

$$
\begin{aligned}
& P_{\mathrm{MH}}(a)=\frac{x \times P_{\mathrm{MH}}(e)}{x+3}, \quad P_{\mathrm{MH}}(b)=\frac{(x+2) \times P_{\mathrm{MH}}(e)}{x+3} \\
& P_{\mathrm{MH}}(c)=\frac{2 P_{\mathrm{MH}}(e)}{x+3}, \quad P_{\mathrm{MH}}(d)=\frac{3 P_{\mathrm{MH}}(e)}{x+3}
\end{aligned}
$$

Since the summation of all tree nodes visiting probability is 1 , the value of $P_{M H}(e)$ can be calculated as follows:

$$
\begin{aligned}
& P_{\mathrm{MH}}(a)+(2 y-2) P_{\mathrm{MH}}(b)+2 P_{\mathrm{MH}}(c)+\left(x^{y}-2\right) P_{\mathrm{MH}}(d) \\
& +\frac{\left(x^{y}-2 x y+x+2 y-2\right)}{x-1} P_{\mathrm{MH}}(e)=1 \\
& \Rightarrow \frac{x \times P_{\mathrm{MH}}(e)}{x+3}+(2 y-2) \frac{(x+2) P_{\mathrm{MH}}(e)}{x+3}+2 \times \frac{2 P_{\mathrm{MH}}(e)}{x+3} \\
& +\left(x^{y}-2\right) \frac{3 P_{\mathrm{MH}}(e)}{x+3}+\frac{\left(x^{y}-2 x y+x+2 y-2\right)}{x-1} P_{\mathrm{MH}}(e)=1 \\
& \Rightarrow \frac{\left(3 x^{y}+2 x y-x+4 y-6\right)}{x+3} P_{\mathrm{MH}}(e)+\frac{\left(x^{y}-2 x y+x+2 y-2\right)}{x-1} P_{\mathrm{MH}}(e)=1 \\
& \Rightarrow P_{\mathrm{MH}}(e)=\frac{(x+3)(x-1)}{4 x^{y+1}-2 x y-4 x+2 y}
\end{aligned}
$$

Other probabilities can be obtained accordingly:

$$
\begin{aligned}
P_{\mathrm{MH}}(a) & =\frac{x(x-1)}{4 x^{y+1}-2 x y-4 x+2 y} \\
P_{\mathrm{MH}}(b) & =\frac{(x+2)(x-1)}{4 x^{y+1}-2 x y-4 x+2 y} \\
P_{\mathrm{MH}}(c) & =\frac{2(x-1)}{4 x^{y+1}-2 x y-4 x+2 y} \\
P_{\mathrm{MH}}(d) & =\frac{3(x-1)}{4 x^{y+1}-2 x y-4 x+2 y}
\end{aligned}
$$




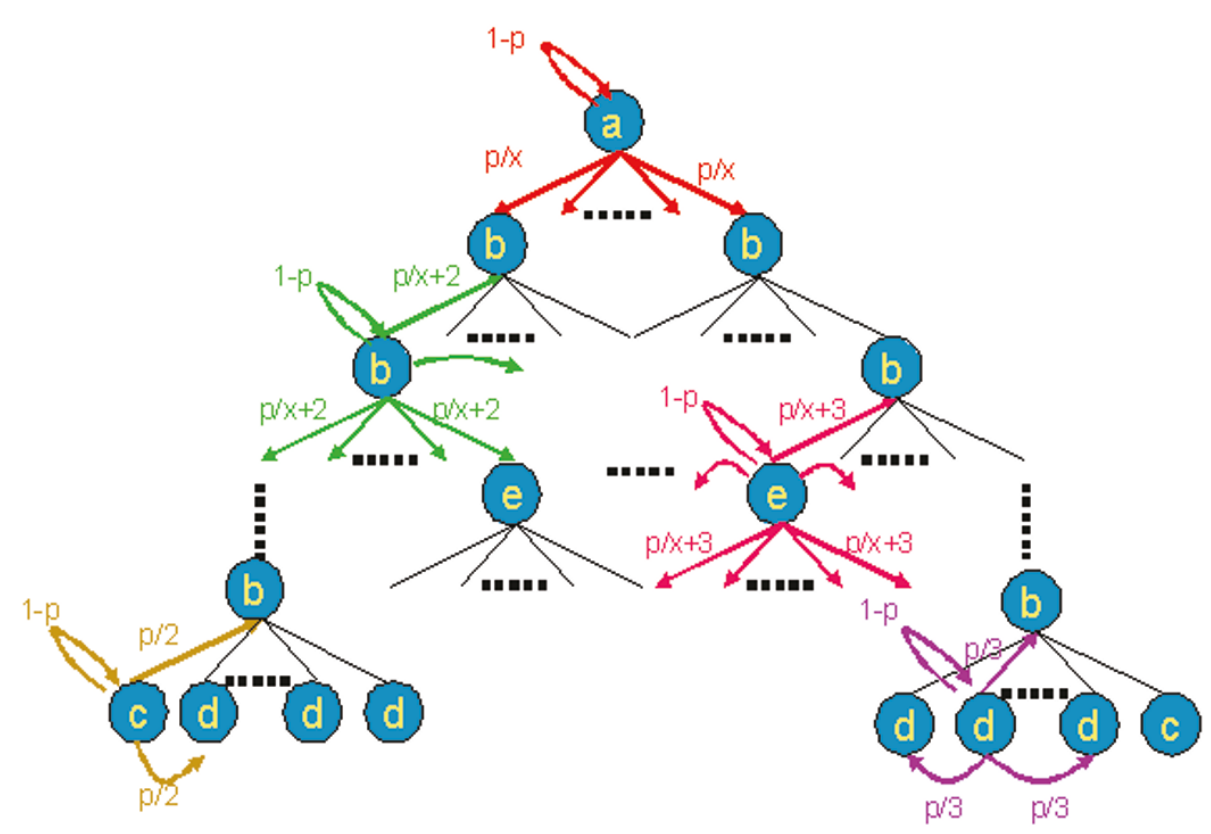

Figure 9 State transition probability in tree topology.

In order to explore more about the tree topology, the p.d.f (probability density function) of the hop count from the BS (root node) to a tree node is investigated. The hop count from the root to each node is displayed in Figure 10. The probability for a given hop count $N$ can be calculated as follows:

$$
p(N)=\left\{\begin{array}{c}
P_{\mathrm{MH}}(a), N=0 \\
2 P_{\mathrm{MH}}(c)+\left(x^{y}-2\right) \times P_{\mathrm{MH}}(d), N=y \\
2 P_{\mathrm{MH}}(b)+\left(x^{y}-2\right) \times P_{\mathrm{MH}}(e), \text { otherwise }
\end{array}\right.
$$

Figures 11 and 12 display the p.d.f of the hop count from the BS in the tree topology with $x=2$ and $x=3$, respectively. The figures demonstrate the characteristic of larger hop counts with higher probabilities (exponential-like behavior), which causes challenge in bandwidth management.

\section{Chessboard topology}

Based on a similar Markovian analysis method, the average visiting probability of MHs at each RS in the chessboard topology can be obtained. The size of the chessboard topology is $(2 n-1) *(2 n-1)$. Let $P_{\mathrm{MH}}(i, j)$ denotes the probability that the $\mathrm{MH}$ is at location $(i, j)$, and $p$ denotes the probability that the MH leaves its current network. The Markov Chain for the chessboard topology is displayed in Figure 13. The value of $P_{\mathrm{MH}}(i, j)$ is the probability that $\mathrm{MH}$ stays at location $(i, j)$ plus the probability that the $\mathrm{MH}$ moves in from neighbor networks. Thus, the value of $P_{\mathrm{MH}}(1,1)$ can be found as follows:

$$
\begin{aligned}
& P_{\mathrm{MH}}(1,1)=P_{\mathrm{MH}}(1,1) \times(1-p)+P_{\mathrm{MH}}(1,2) \times \frac{p}{3}+P_{\mathrm{MH}}(2,1) \times \frac{p}{3} \\
& =\frac{P_{\mathrm{MH}}(1,2)}{3}+\frac{P_{\mathrm{MH}}(2,1)}{3}
\end{aligned}
$$

$P_{\mathrm{MH}}(1,2), P_{\mathrm{MH}}(1,3), \ldots$, etc., can also be expressed by similar equations:

$P_{\mathrm{MH}}(1,2)=\frac{P_{\mathrm{MH}}(1,1)}{2}+\frac{P_{\mathrm{MH}}(1,3)}{3}+\frac{P_{\mathrm{MH}}(2,2)}{3}, P_{\mathrm{MH}}(1,3)=\frac{P_{\mathrm{MH}}(1,2)}{3}+\frac{P_{\mathrm{MH}}(1,4)}{3}+\frac{P_{\mathrm{MH}}(2,3)}{4}, \ldots$, etc.

and $\sum_{i=1}^{2 n-1} \sum_{j=1}^{2 n-1} P_{\mathrm{MH}}(i, j)=1$

Similar to the Markovian analysis in [37], the relationship of visiting probability among nodes' given in the following equations.

$$
\left\{\begin{array}{l}
P_{\mathrm{MH}}(1,1)=P_{\mathrm{MH}}(1,2 n-1)=P_{\mathrm{MH}}(2 n-1,1) \\
\quad=P_{\mathrm{MH}}(2 n-1,2 n-1)=\frac{1}{2} \times \frac{1}{4 n^{2}-6 n+2} \\
P_{\mathrm{MH}}(1, a)=P_{\mathrm{MH}}(2 n-1, a)=P_{\mathrm{MH}}(a, 1) \\
\quad=P_{\mathrm{MH}}(a, 2 n-1)=\frac{3}{4} \times \frac{1}{4 n^{2}-6 n+2} \\
P_{\mathrm{MH}}(a, b)=\frac{1}{4 n^{2}-6 n+2}
\end{array}\right.
$$

where $a, b \in\{2,3, \ldots 2 n-2\}$.

There are three categories of node in the above equations. The first category includes nodes on four corners 

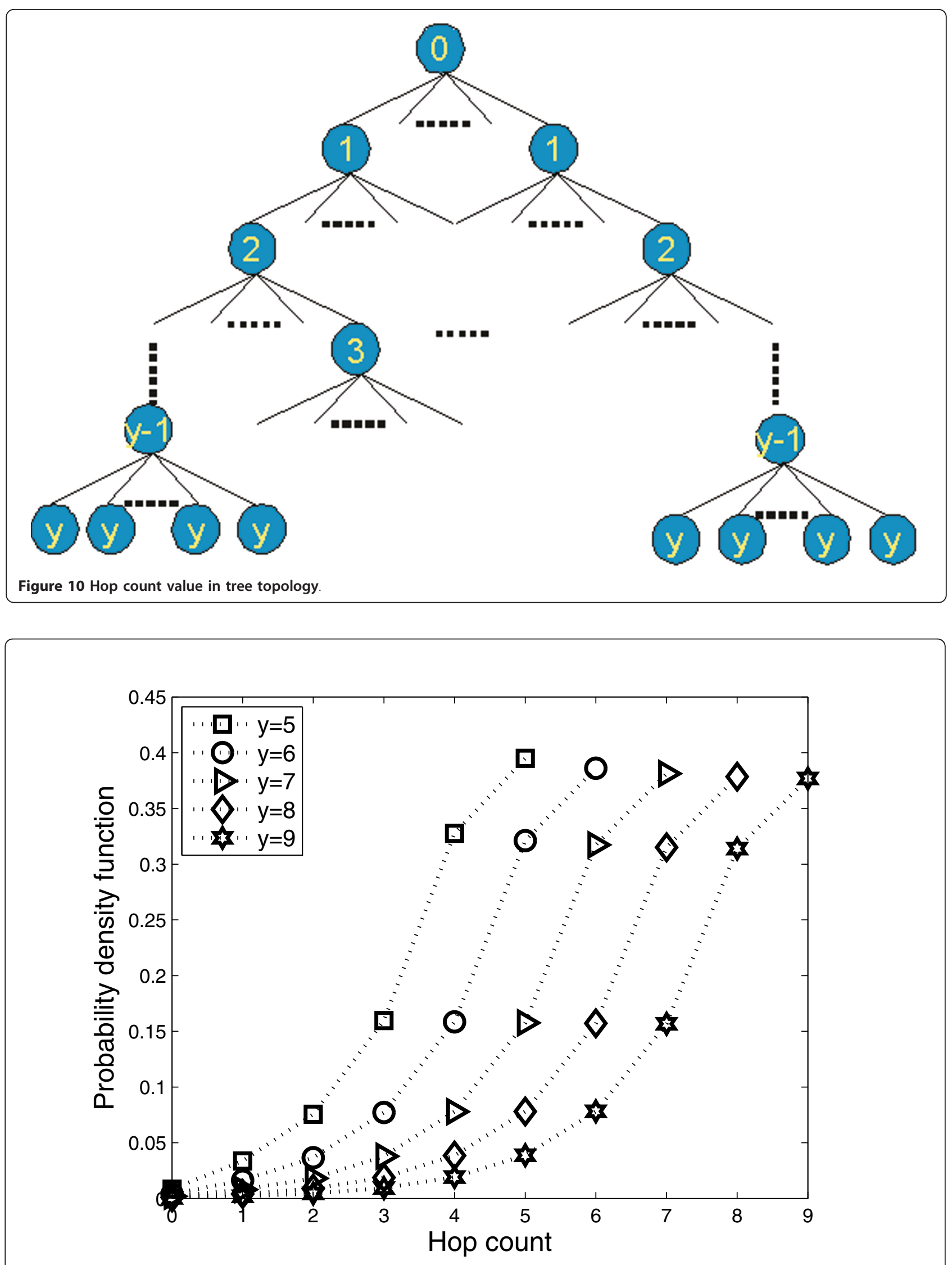

Figure 11 The p.d.f for hop count (Tree degree $=2$ ). 


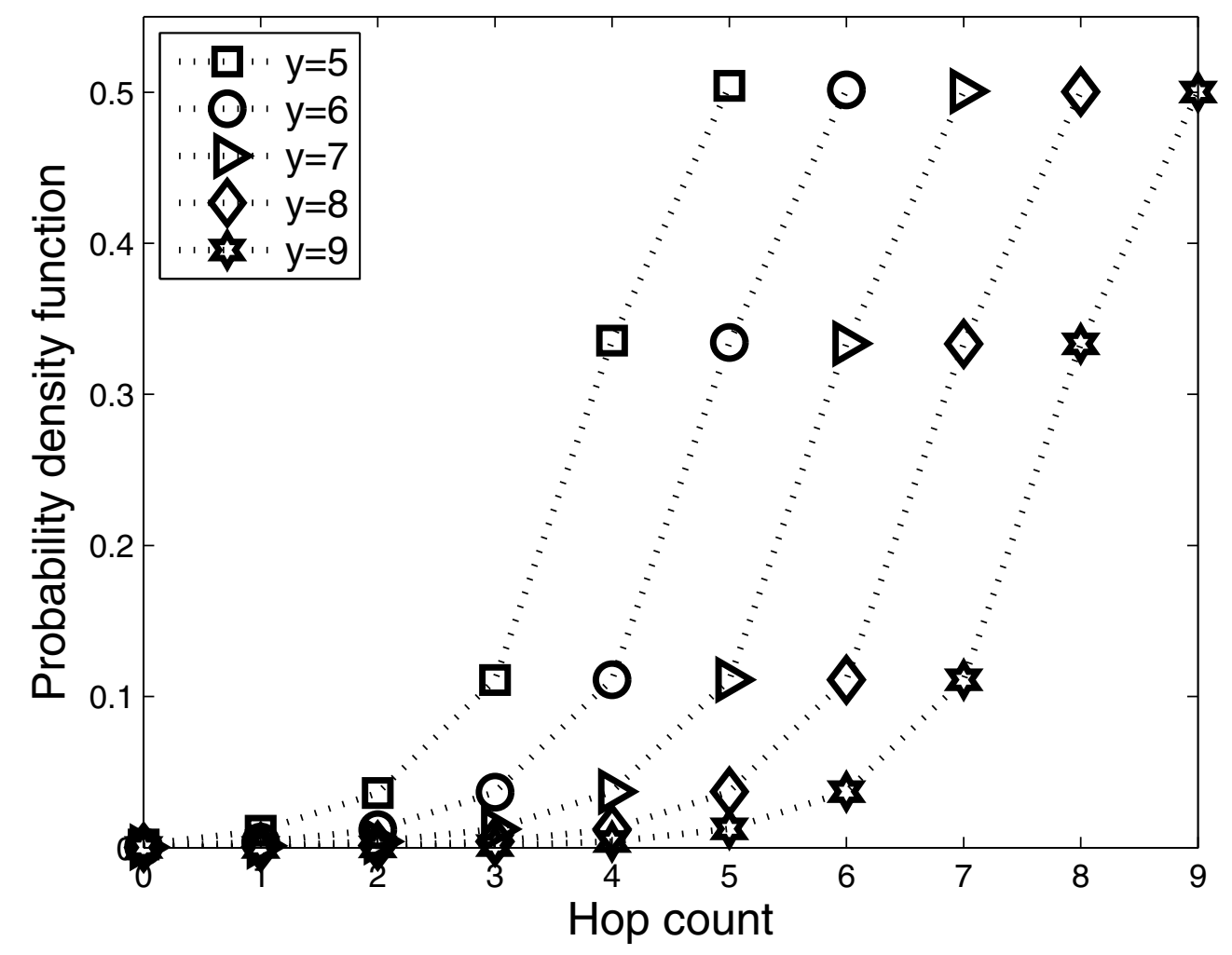

Figure 12 The p.d.f for hop count (Tree degree $=3$ ).

of the chessboard topology. Each of this kind of nodes comes with two neighbors. The probability of a $\mathrm{MH}$ visiting a neighbor is $\frac{1}{2} * \frac{1}{4 n^{2}-6 n+2}$. The second category includes nodes on edges of the chessboard topology. Each of this kind of nodes comes with three neighbors. The probability of a $\mathrm{MH}$ visiting a neighbor is $\frac{3}{4} * \frac{1}{4 n^{2}-6 n+2}$. All the other nodes belong to the third category. Each of this kind of nodes comes with four neighbors. The probability of a $\mathrm{MH}$ visiting a neighbor is $\frac{1}{4 n^{2}-6 n+2}$.

Figure 14 displays the p.d.f of the hop count from the BS (the upper leftmost node) to a node in the chessboard topology with different sizes $(n=3-6)$. The figure demonstrates a Normal distribution-like behavior, which is more appropriate for bandwidth management in comparison with the tree topology. Therefore, the chessboard topology is the main focus in the simulation study.

\section{Simulation environment}

Simulation study has been conducted to evaluate the performance of Zone-based bandwidth management. The IEEE 802.16-MR network is an $11 \times 11$ chessboard topology as the one in Figure 4. The BS is located at the upper leftmost corner, and the $\mathrm{CN}$ is outside the network. Link capacity of the network is 20 Mbps. Parameters used in the simulation are displayed in Table 2.

To get more information on MHs movement, the simulation also designed different properties of mobility distribution. Considering MH movement behavior, there are four proposed experiment of mobility distribution: such as $M D \_E q u a l, M D_{-}$CloseToCenter, MD_CloseToBS, and $M D \_$AwayFromBS. The first mobility distribution $M D \_$Equal has equal probability in each direction. The others have $80 \%$ probability to move to the center of Mesh, the BS, and opposite of the BS, respectively. The p.d.f of the hop count for the four mobility distributions is displayed in Figures 15, 16, 17 and 18.

In MD_Equal, each MH appears at each RS node as the uniform distribution. Since the topology is the 


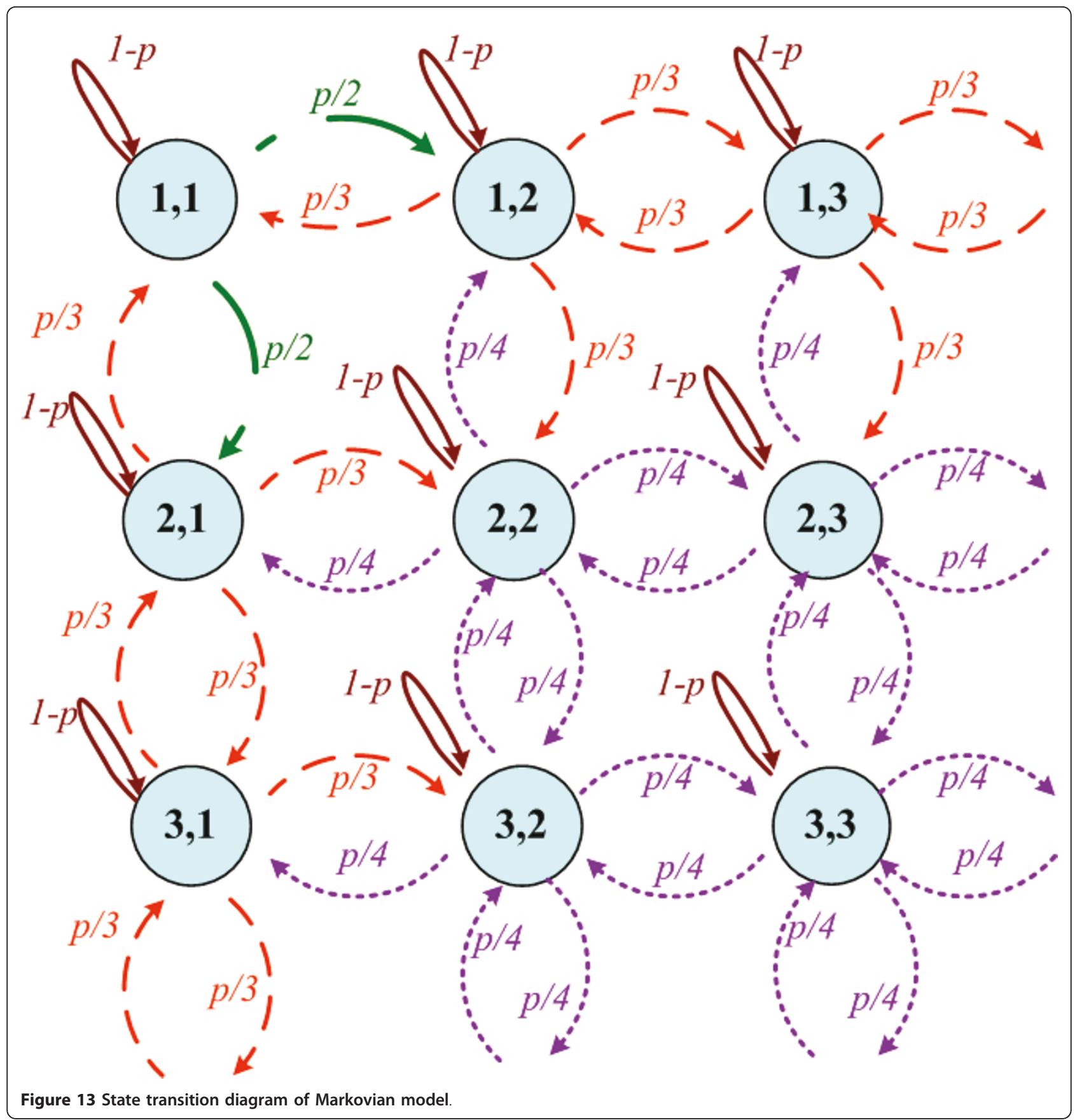

symmetrical Mesh network, the hop count of 11 has maximum number of RSs in Figure 15. The curve is similar to normal distribution so the higher ratio is near the middle hop count in the topology.

In MD_CloseToCenter, MHs have higher mobility probability to the center RS. The center of RSs get higher visiting probability, hence the peak curve of occurrence density ratio also appears near the middle hop count in Figure 16.
In MD_CloseToBS, the MH moves toward the BS with much higher probability, which results in higher visiting probability for the nodes near the BS as shown in Figure 17. The behavior of $M D_{-}$AwayFromBS is the opposite of $M D \_C l o s e T o B S$. The visiting ratio is opposite to the case of $M D \_C l o s e T o B S$, which results in higher probability for the nodes farther from the BS as shown in Figure 18. 


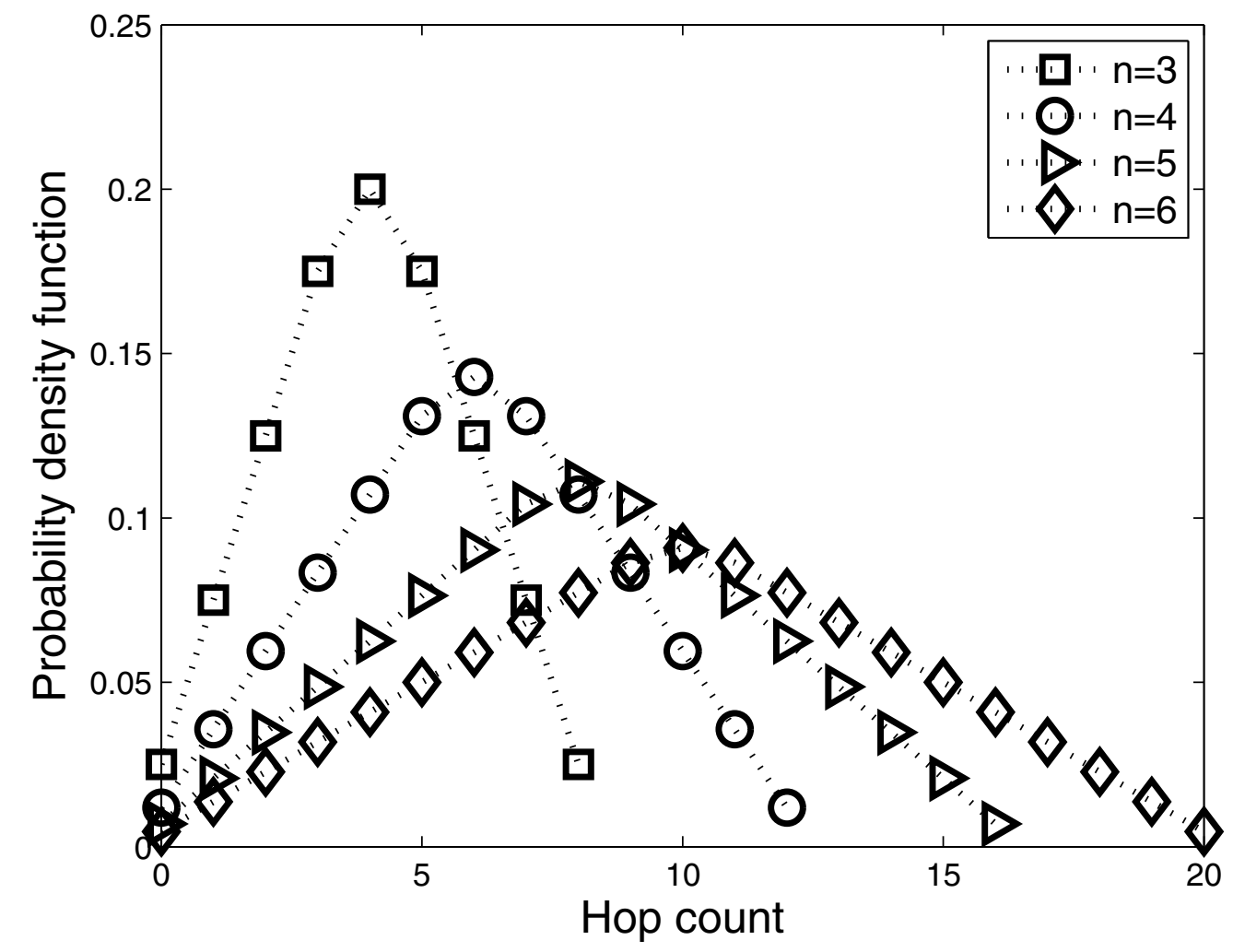

Figure 14 The p.d.f for hop count.

\section{Simulation criteria}

Some criteria are defined for performance evaluation.

(1) Bandwidth Allocation is defined in the unit of hop count, since the flows in the simulation are all UGS flows with same data rate of BW.

(2) Average Satisfaction is the average ratio of allocated bandwidth over required bandwidth. Standard Deviation of Satisfaction is used to evaluate the fluctuation of the allocated bandwidth.

Table 2 Simulation parameters.

\begin{tabular}{cc}
\hline Description & Value \\
\hline Topology size & $11^{* 11}$ Mesh \\
\hline System bandwidth & $20 \mathrm{Mbps}$ \\
\hline Time frame & $10 \mathrm{~ms}$ \\
\hline \# of Minislot in time frame & 500 \\
\hline Simulation time & $100 \mathrm{~s}$ \\
\hline Flow type & UGS \\
\hline Flow rate & $3.65 \mathrm{Kbps}$ \\
\hline Flow life time & $50 \mathrm{~s}$ \\
\hline Number of flow & 100 to 1000
\end{tabular}

(3) Bandwidth Re-allocation Ratio is the ratio of the case that bandwidth re-allocation is triggered over the total number of handoff.

(4) Bandwidth Utilization is the actual utilization rate of system resources.

(5) Handoff Call Degradation Ratio is the ratio of the case that the required bandwidth cannot be met after $\mathrm{MH}$ handoff.

(6) New Call Blocking Probability is the rate of new MHs failing to enter the 802.16-MR network. It can be obtained by: Number of tries over number of MHs successfully entering the system. If a $\mathrm{MH}$ fails, it will be offered the opportunity to try again in the next round.

\section{Simulation results}

\section{Average reserved hop count}

To know the relation of $S_{-} T H$ and $L$ on Bandwidth Allocation with four kinds of mobility distribution results are shown in Figures 19, 20, 21 and 22. To reach the higher Satisfaction, it also needs to reserve more bandwidth in larger zone size $L$. However, if we only want to achieve $90 \%$ or lower Satisfaction, it 


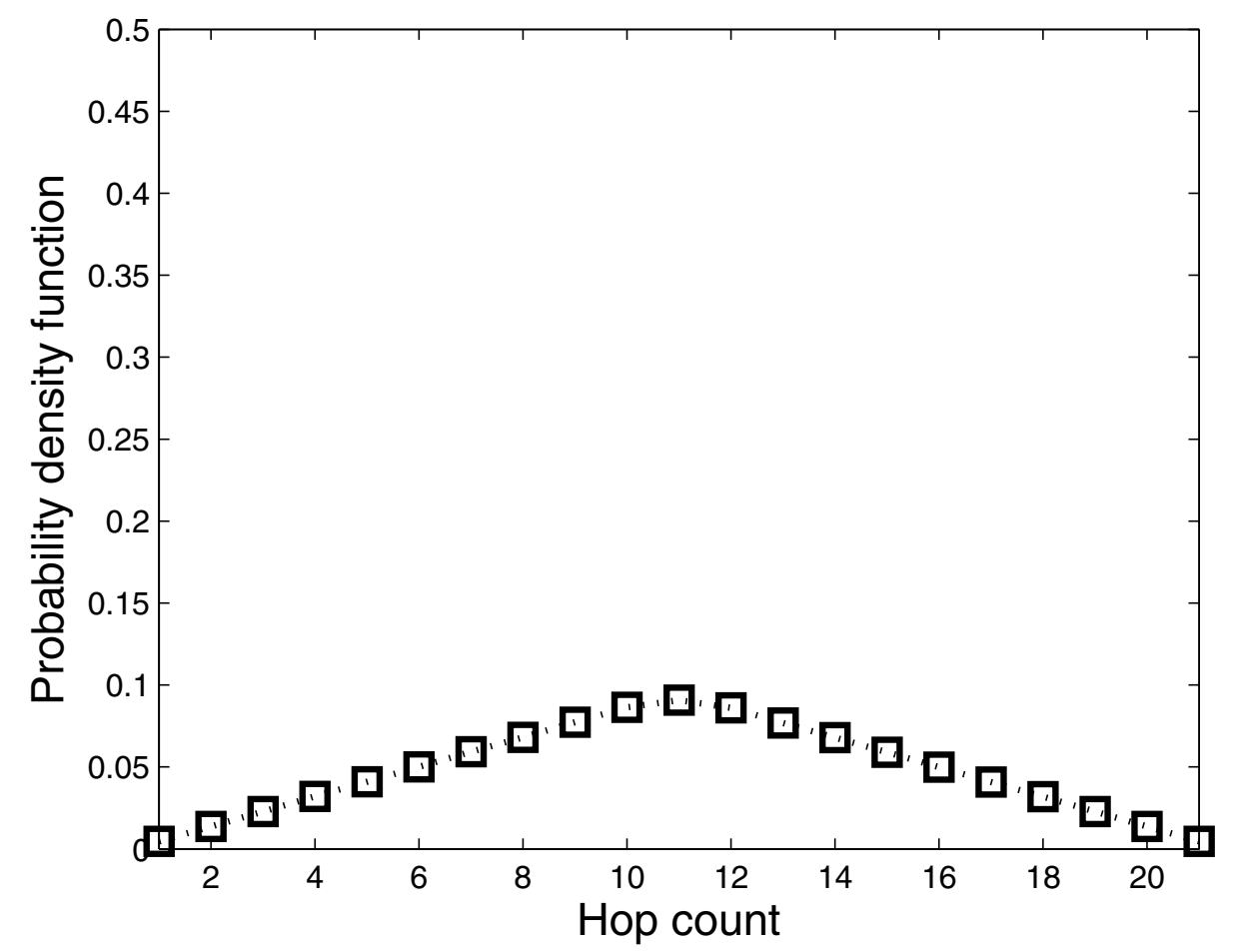

Figure 15 The p.d.f for hop count in MD_Equal.

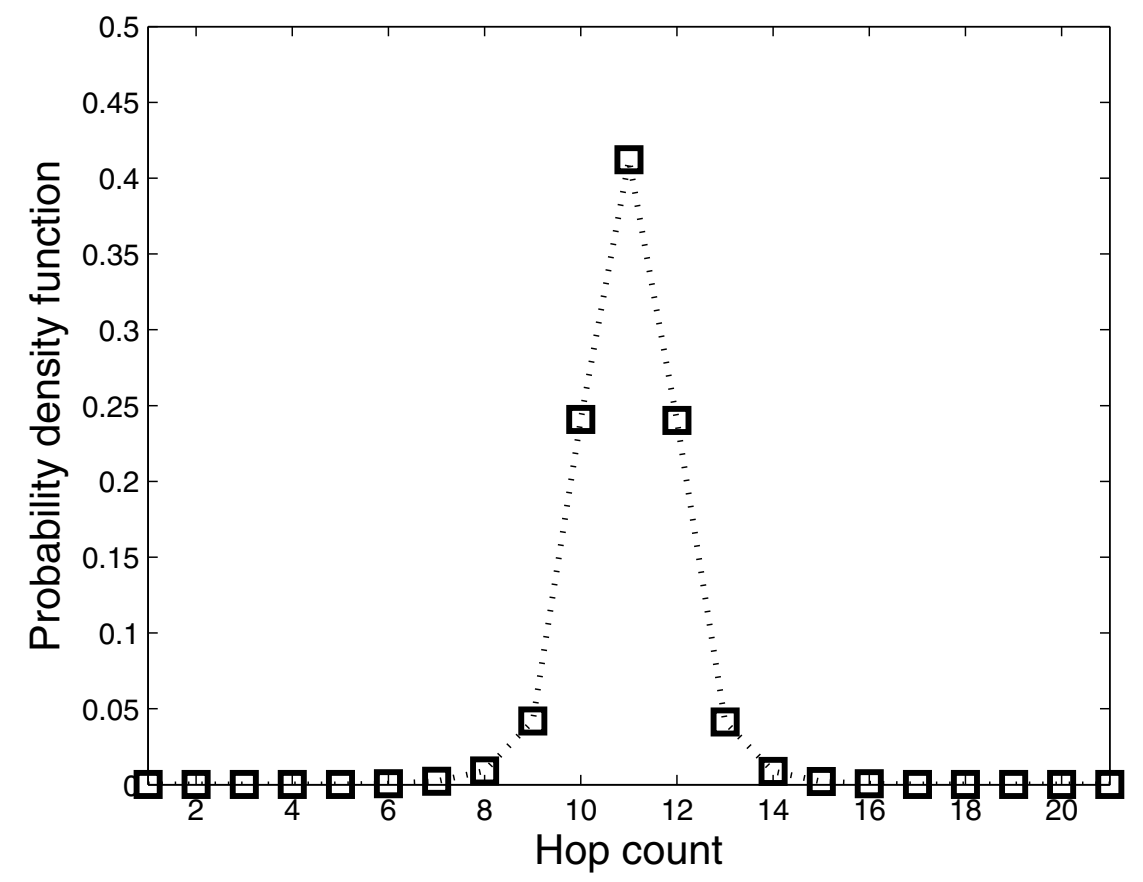

Figure 16 The p.d.f for hop count in MD_CloseToCenter. 


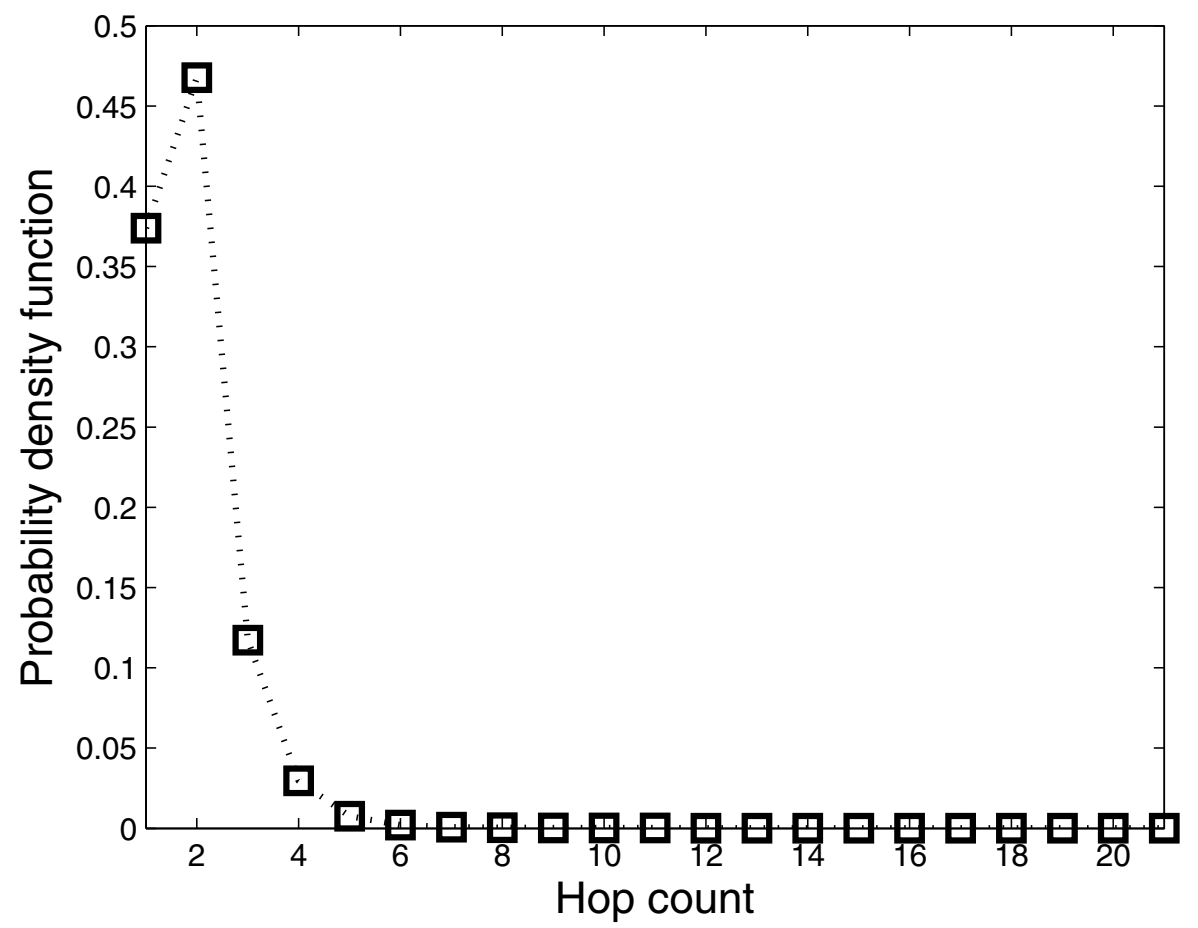

Figure 17 The p.d.f for hop count in MD_CloseToBS.

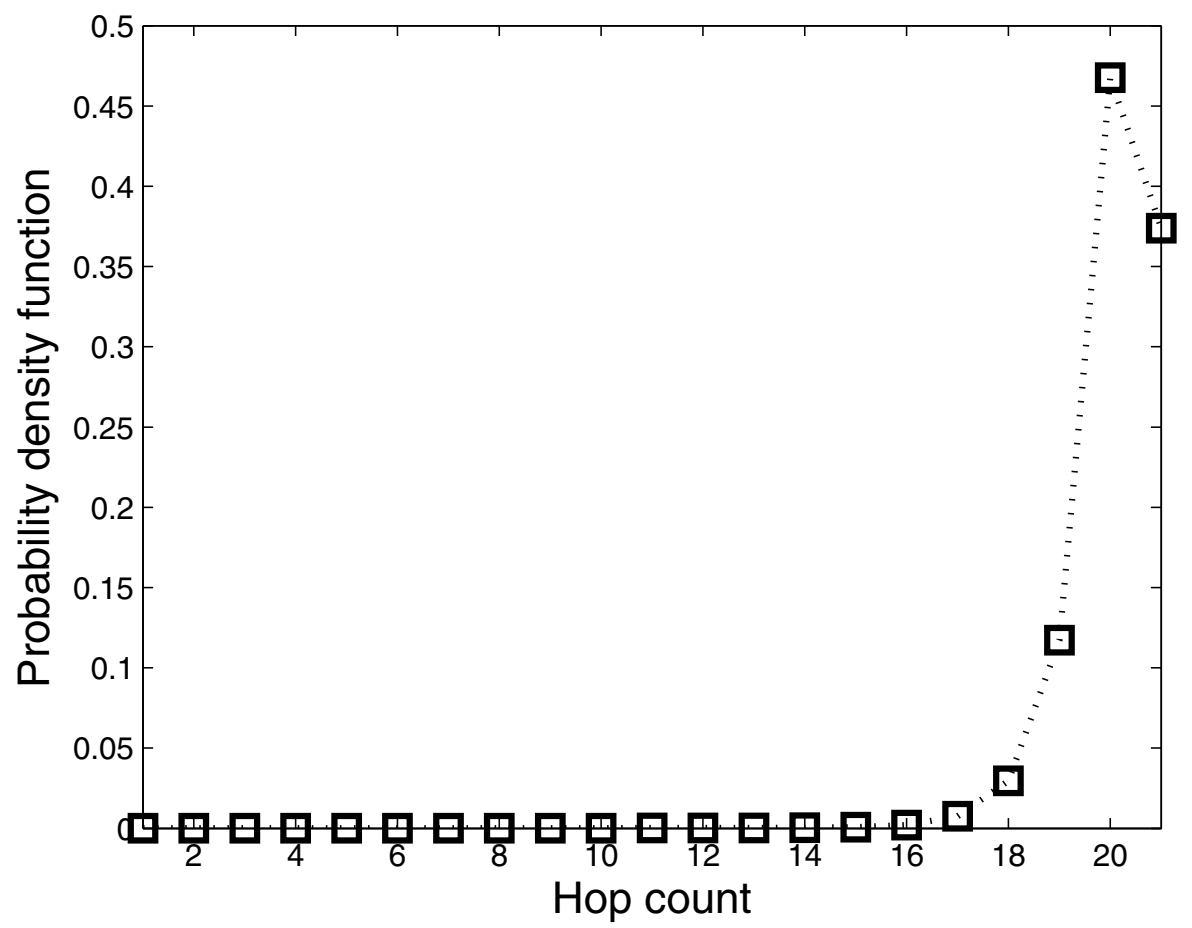

Figure 18 The p.d.f for hop count in MD_AwayFromBS. 


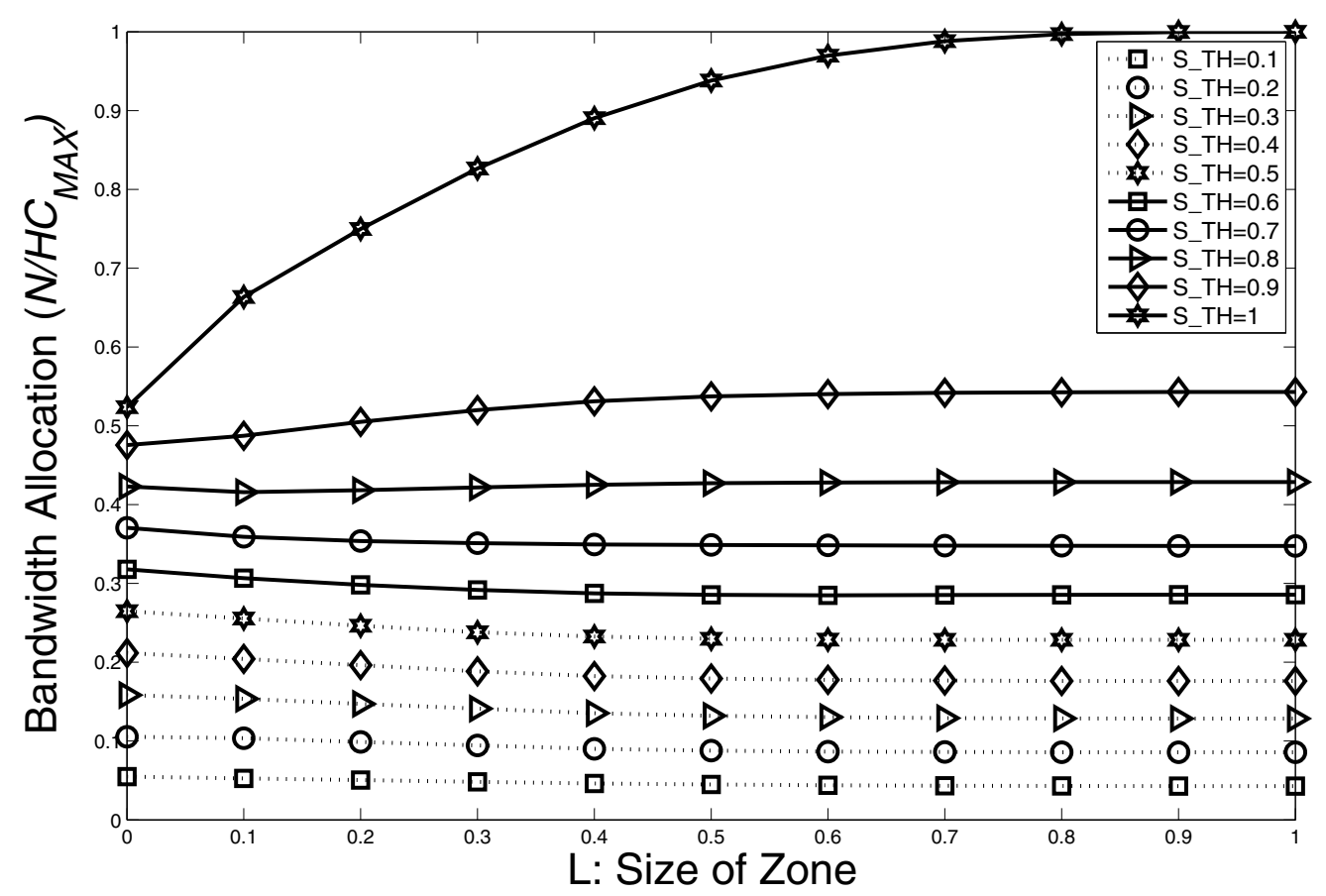

Figure 19 Average bandwidth allocation in MD_Equal.

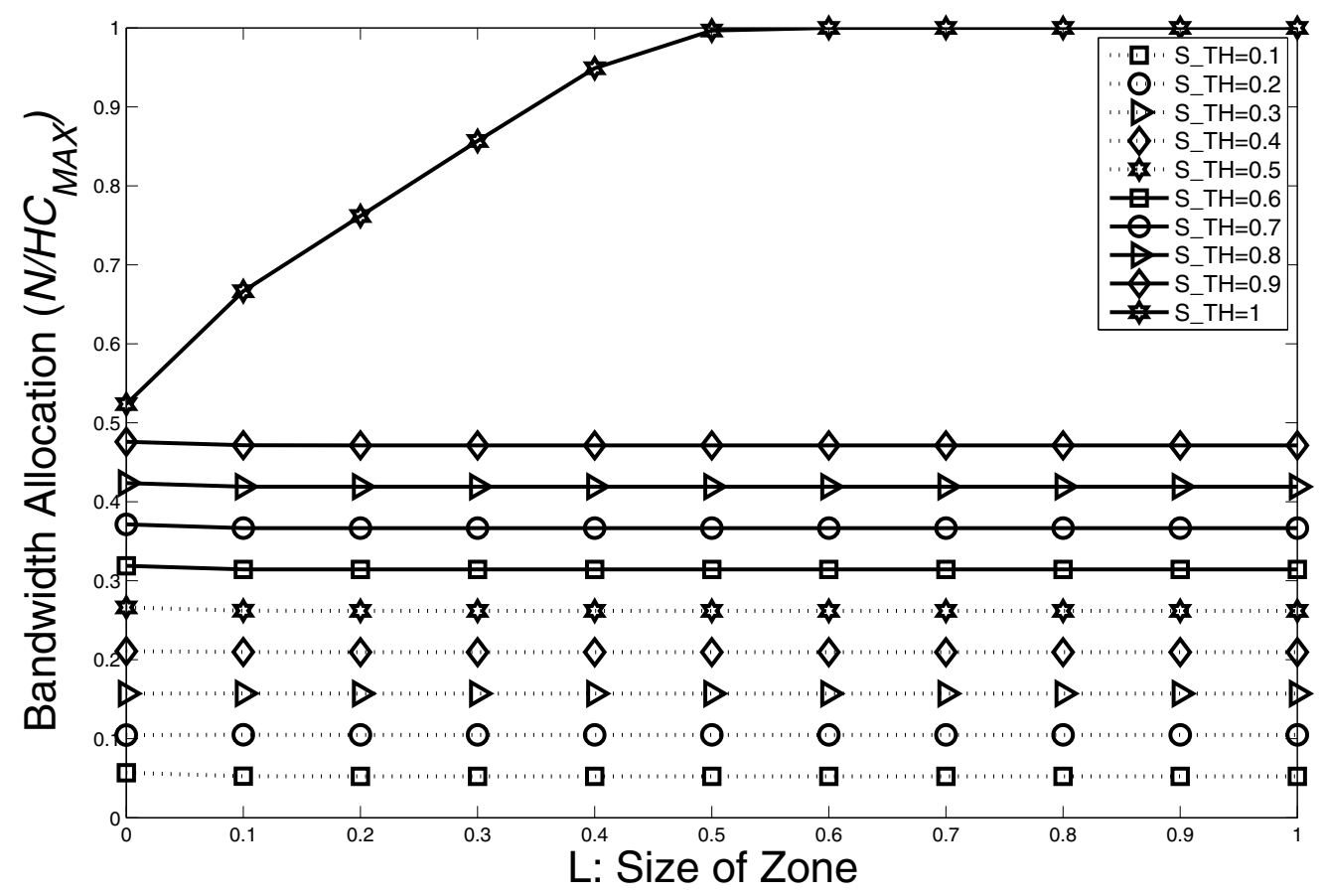

Figure 20 Average bandwidth allocation in MD_CloseToCenter. 

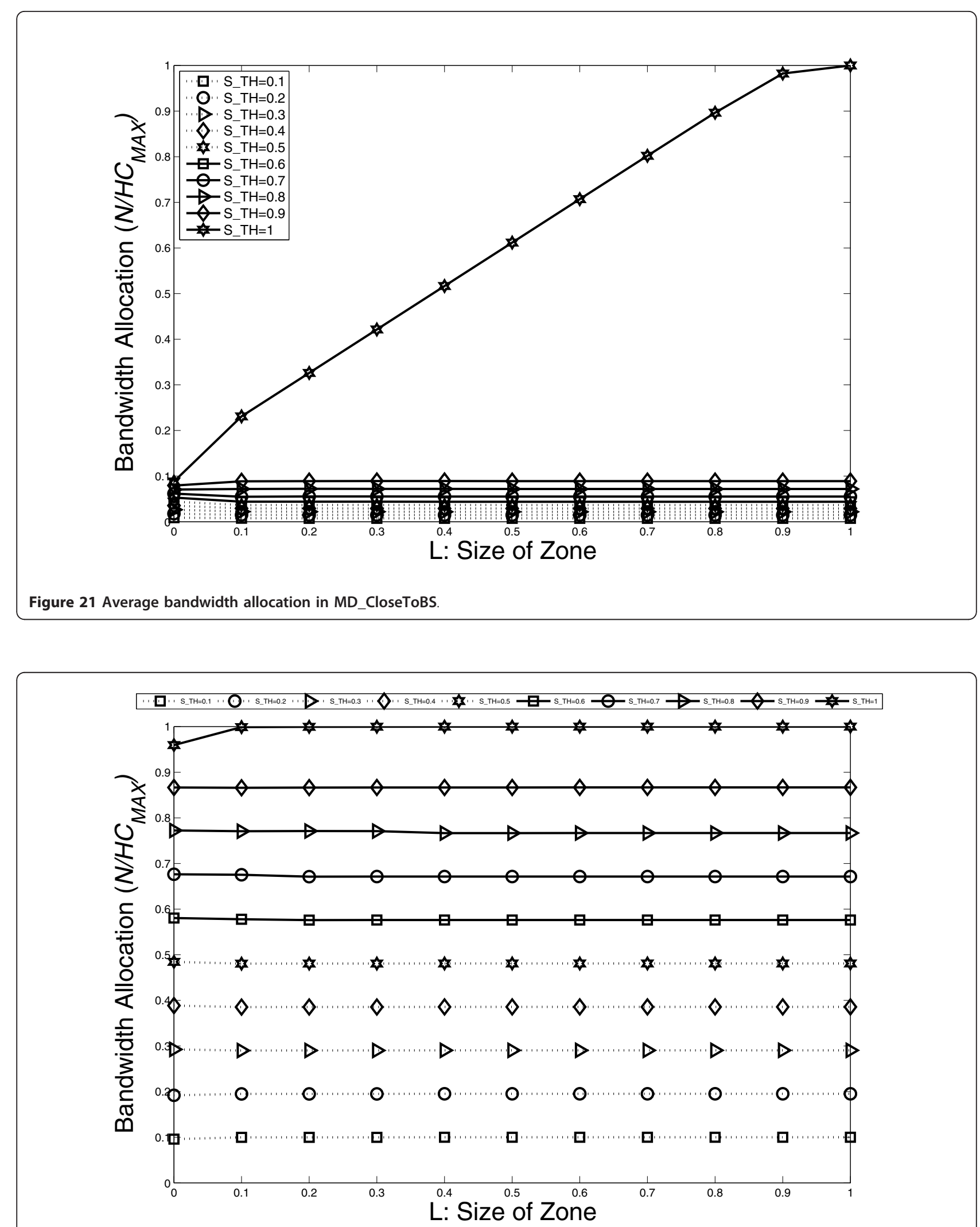

Figure 22 Average bandwidth allocation in MD_AwayFromBS. 


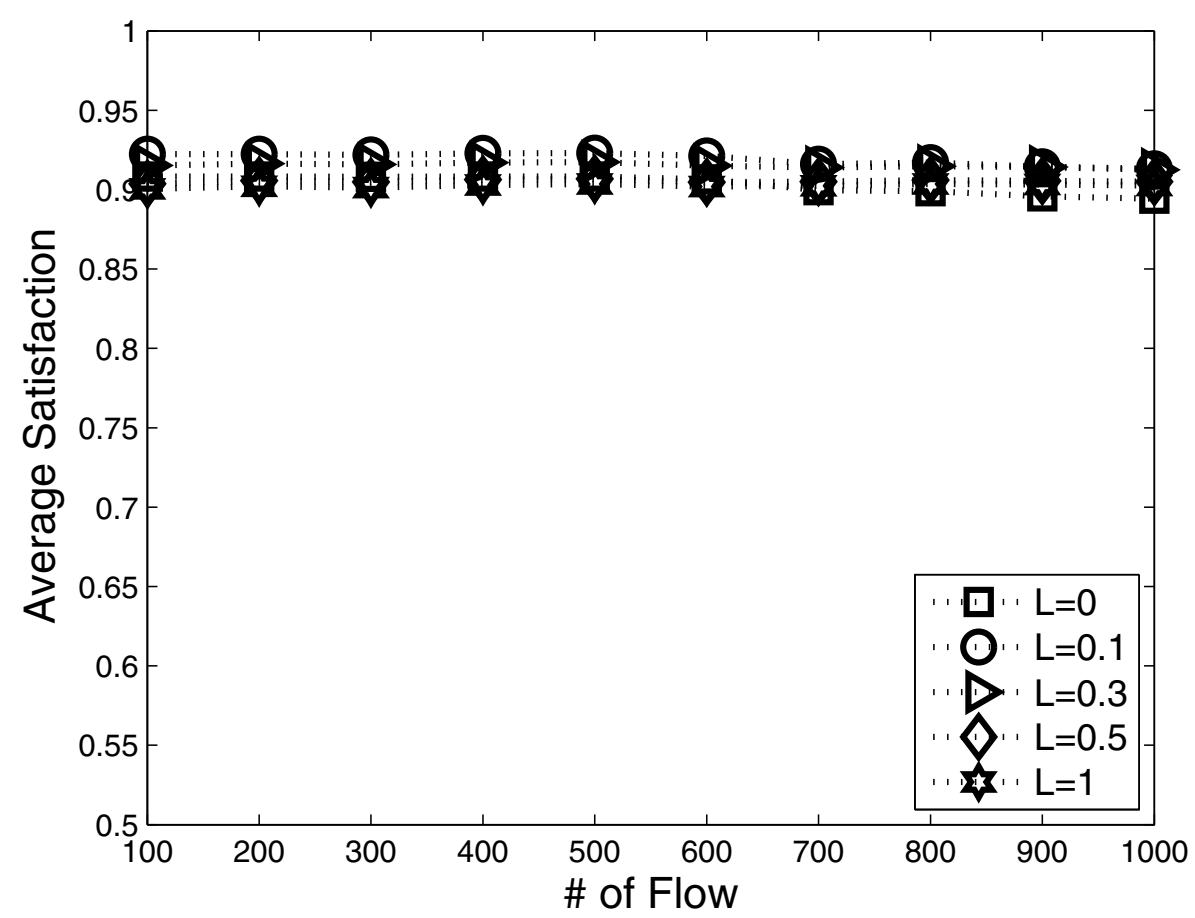

Figure 23 Average satisfaction in MD_Equal with $S \_T H=0.9$

might only need to reserve lower than $60 \%$ hop count expect the case of $M D \_$AwayFromBS in 802.16-MR network. For this reason, it might efficiently use the system bandwidth and obviously reduce MH's bandwidth request. In case of MD_Equal, the hop count could only be allocated under $60 \%$ when $S \_T H \leq 0.9$. When $S_{-} T H=1$, it should allocate more bandwidth with higher hop count when $L$ increase gradually; in $M D \_C l o s e T o C e n t e r$ Case, it is similar to MD_Equal; in MD_CloseToBS Case, MHs have higher moving rate to $\mathrm{BS}$ so it only needs to prepare a little reserved bandwidth when $S_{-} T H<1$. The final case of $M D_{-} A$ wayFromBS, the values of $S_{-} T H$ and Bandwidth Allocation are almost the same and increasing simultaneously.

\section{Average satisfaction rate and standard deviation}

Simulation result of Average Satisfaction Rate is displayed in Figures 23, 24, 25 and 26, the trend of MHs' moving behaviors have been identified so those demonstrate the user requirement bandwidth $\left(S_{-} T H=0.9\right)$ can be achieved by the proposed Zone-based scheme in each kind of mobility distribution. As shown in Figures 27, 28, 29 and 30, all cases of Standard Deviation of Satisfaction do not exceed 0.15. The variation of Satisfaction was controlled extremely well. Thus can be seen, our proposed Zone-based scheme can satisfy MH's requirement and achieve lower variation for $\mathrm{MH}$ mobility. It might a good idea to reserve bandwidth resource depend on user's Satisfaction.

\section{Re-allocated bandwidth, utilization, and blocking ratio}

Considering MHs handoff overhead, the parameter of Bandwidth Re-allocation Ratio can represent MHs' handoff cost. In Figures 31, 32 and 33 are Bandwidth Re-allocation Ratio with different traffic load, all figures decrease exponential with zone size $L$. When adopting our proposed Zone-based scheme, the higher zone size $L$ can reduce the frequency of BW request and signal cost, and it can also effectively reduce the BS overhead. Consequently, the larger zone size $L$ can provide higher quality and seamless handoff for MHs within zone area and decrease number of bandwidth re-allocation procedure in BS. Since the four mobility distribution have the same specific, we only show the case of MD_Equal in Utilization, New Call Blocking Probability, and Handoff Call Degradation Ratio (Figures 34, 35 and 36) with $S_{-} T H=0.9$. In Figure 34, the Utilization increases based on \# of flow and the Utilization saturated when flows are upper than 800, larger $L$ has lower bandwidth utilization due to bandwidth reservation to support Intra-Zone handoff. Considering MHs' blocking and dropping ratio, the New Call Blocking Probability and the Handoff Call Degradation Ratio are increasing when 


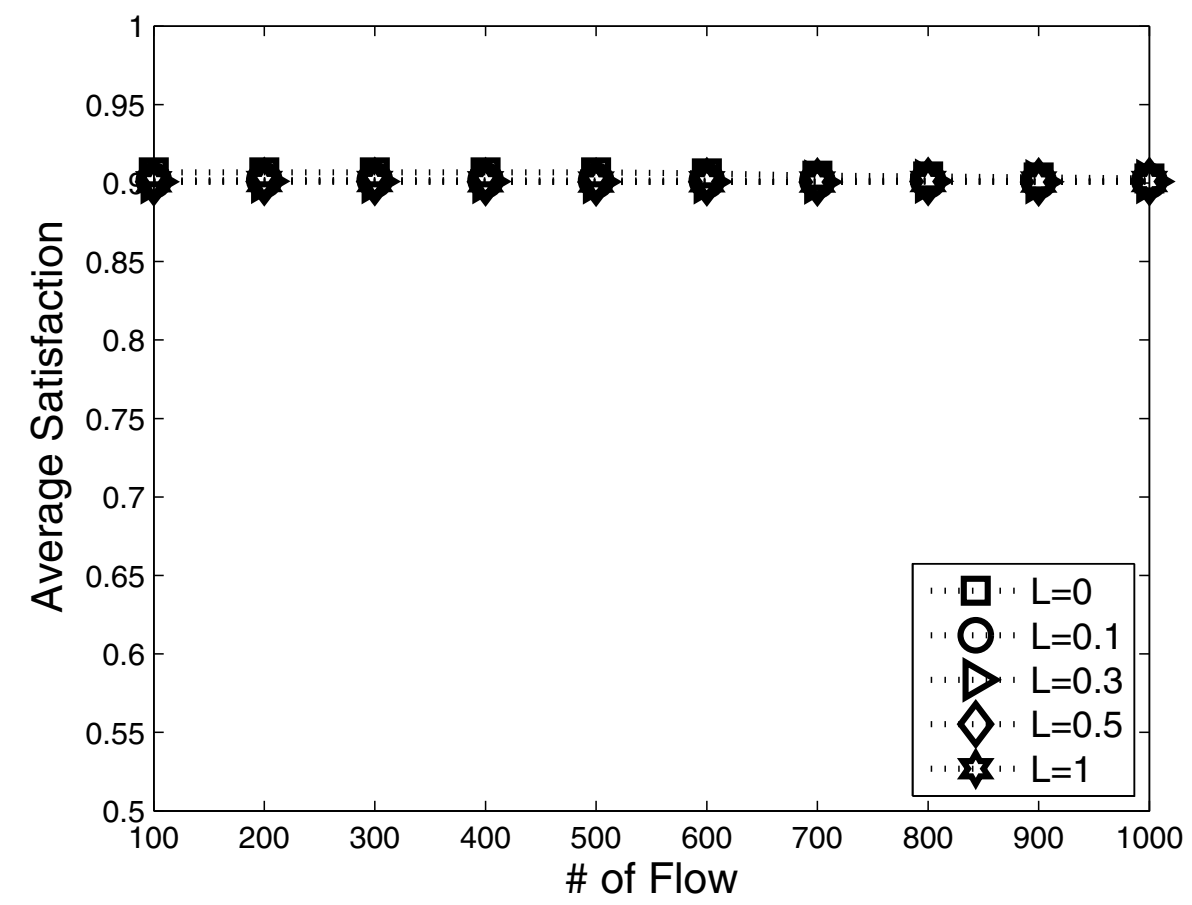

Figure 24 Average satisfaction in MD_Close To Center with $S_{-} T H=0.9$.

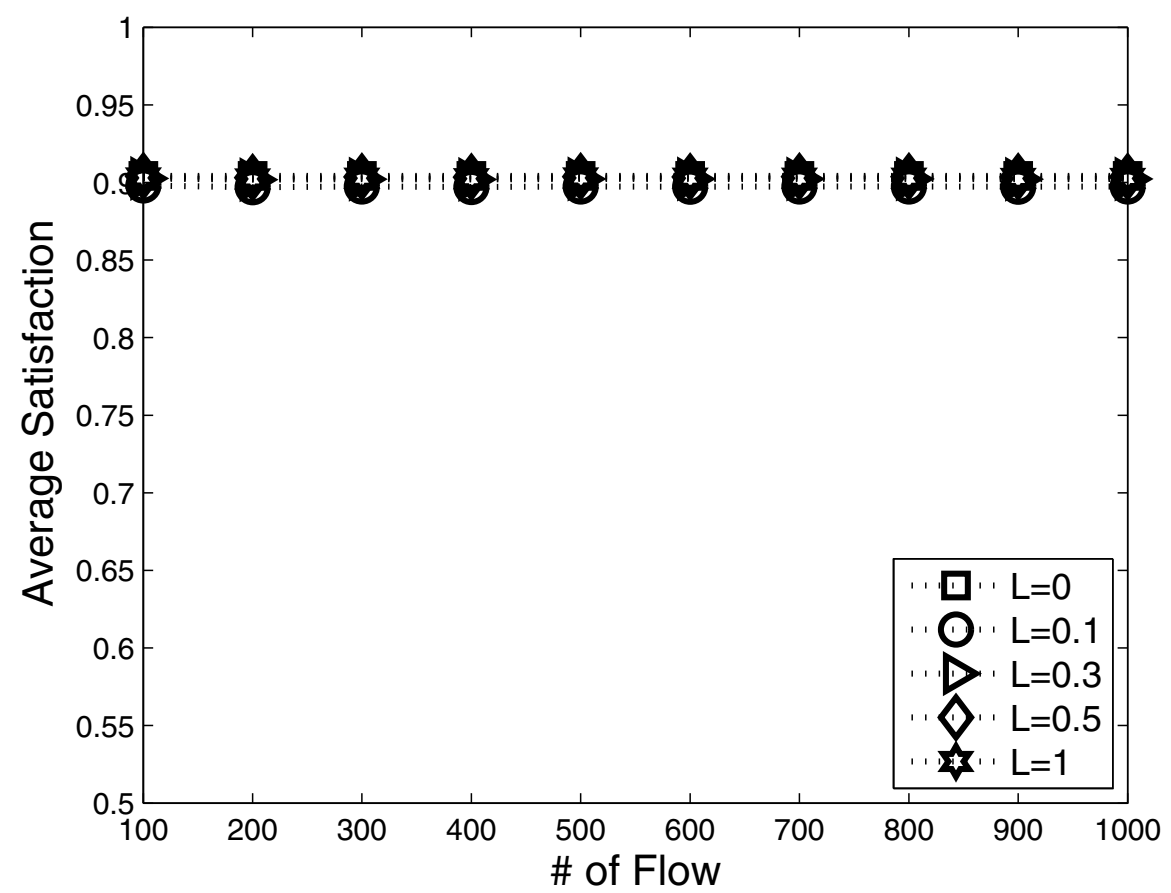

Figure 25 Average satisfaction in MD_Close To BS with $S_{-} T H=0.9$ 


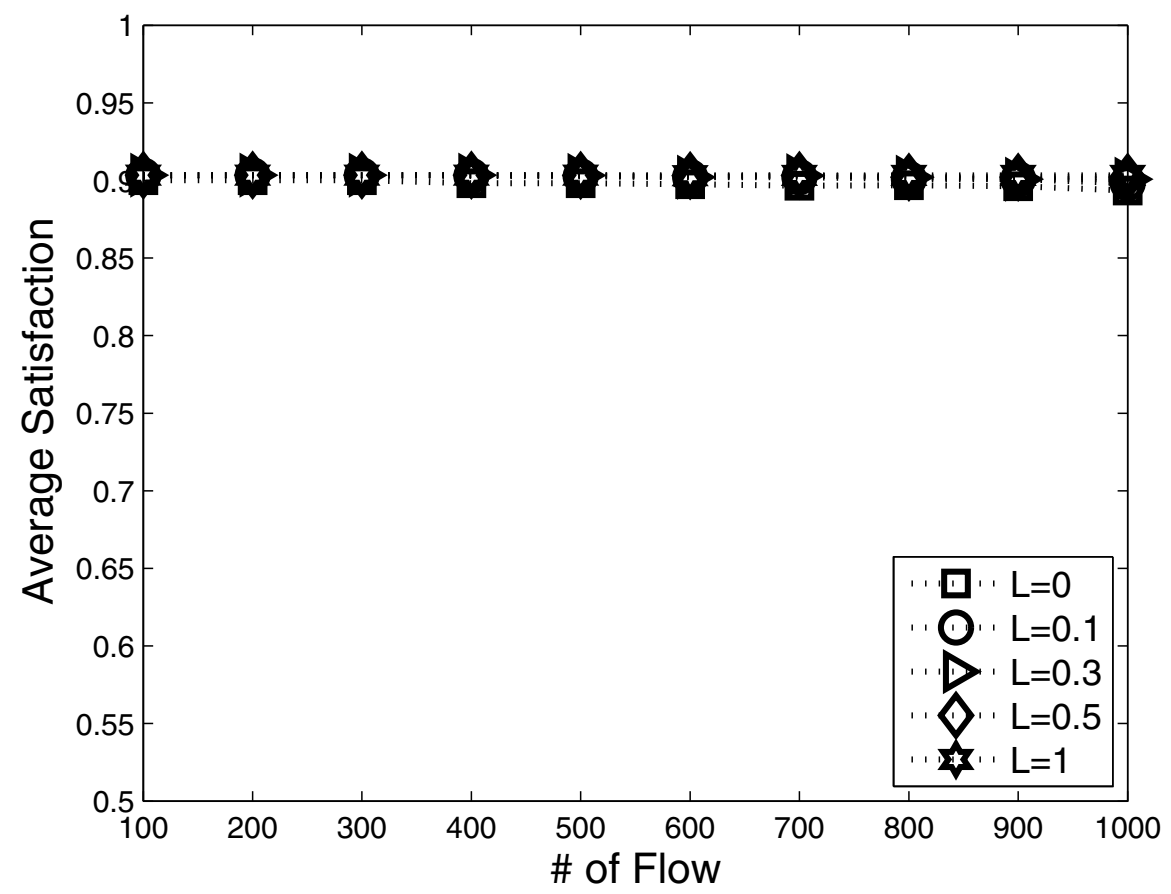

Figure 26 Average satisfaction in MD_AwayFromBS with $S \_T H=0.9$.

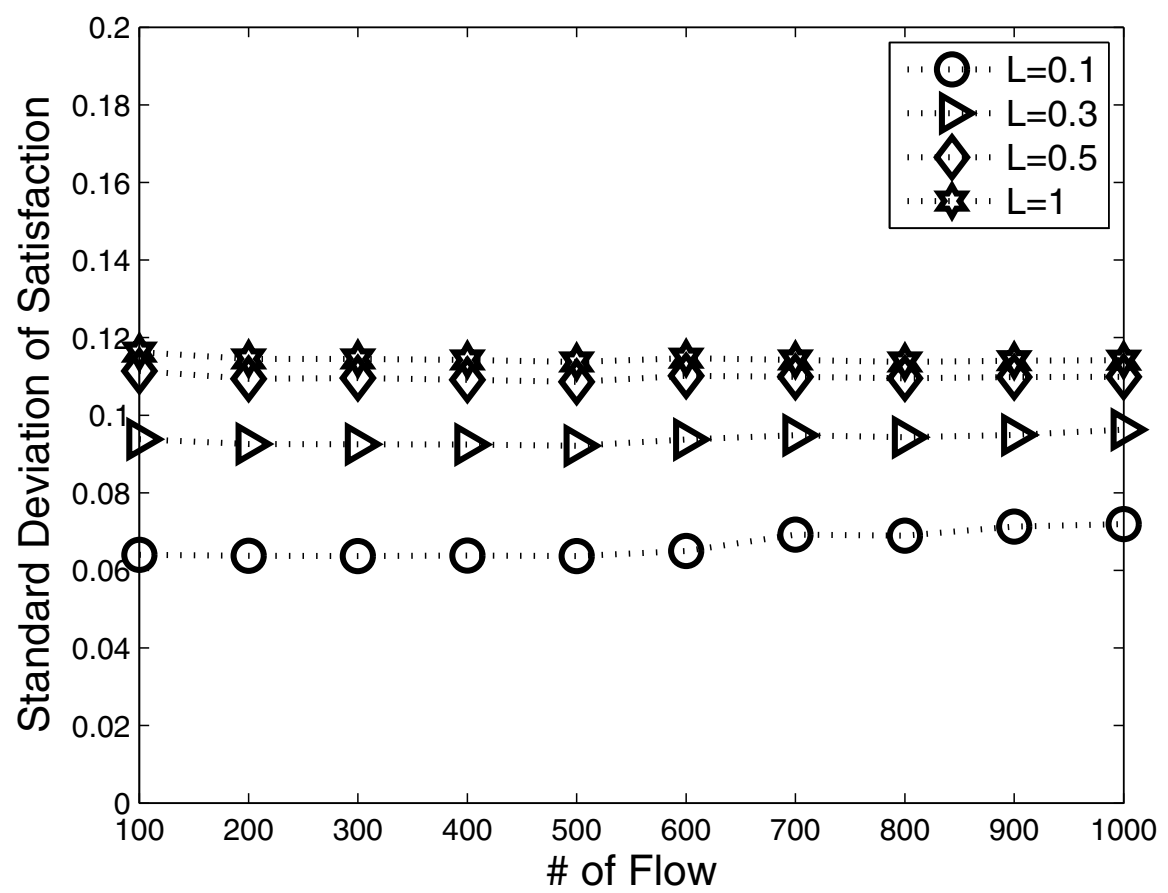

Figure 27 Standard deviation of satisfaction in MD_Equal with $S_{-} T H=0.9$ 


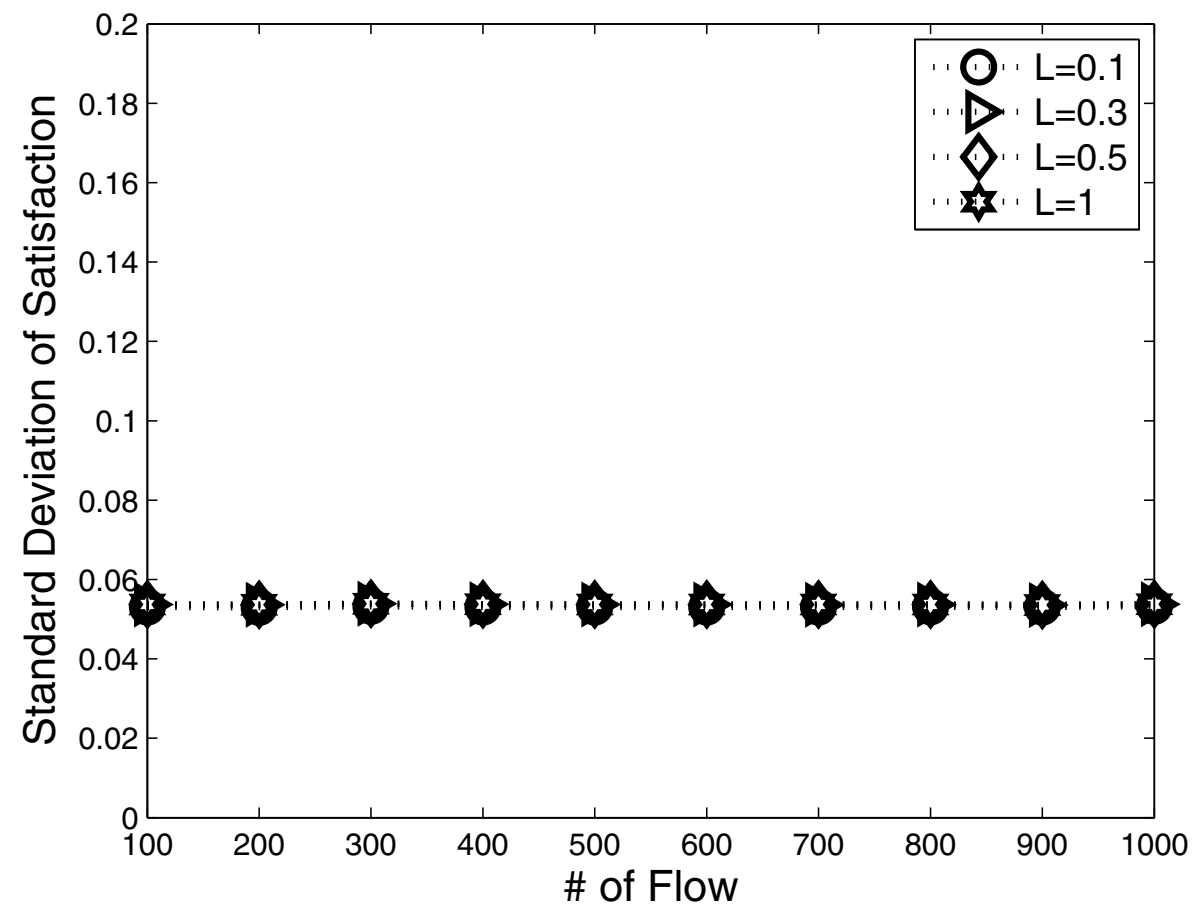

Figure 28 Standard deviation of satisfaction in MD_CloseToCenter with $S \_T H=0.9$.

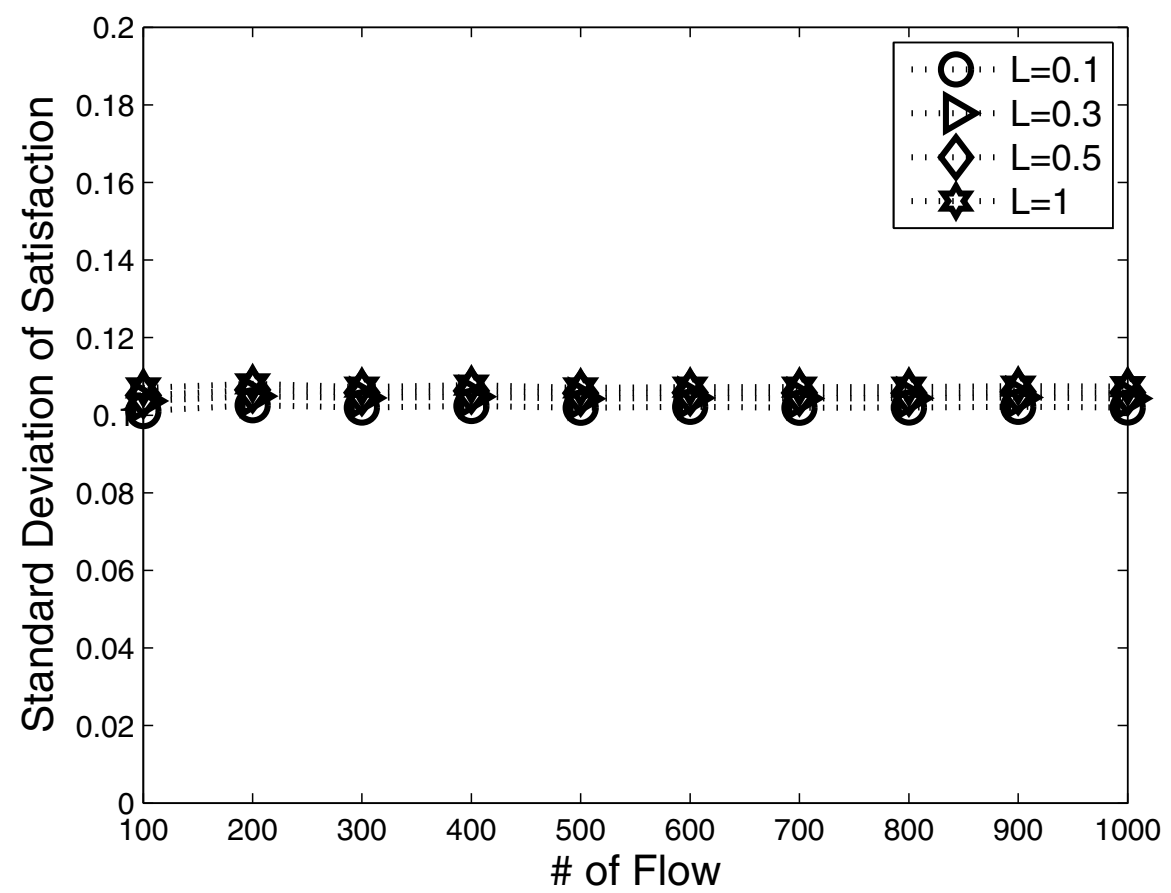

Figure 29 Standard deviation of satisfaction in MD_CloseToBS with $S \_T H=0.9$. 


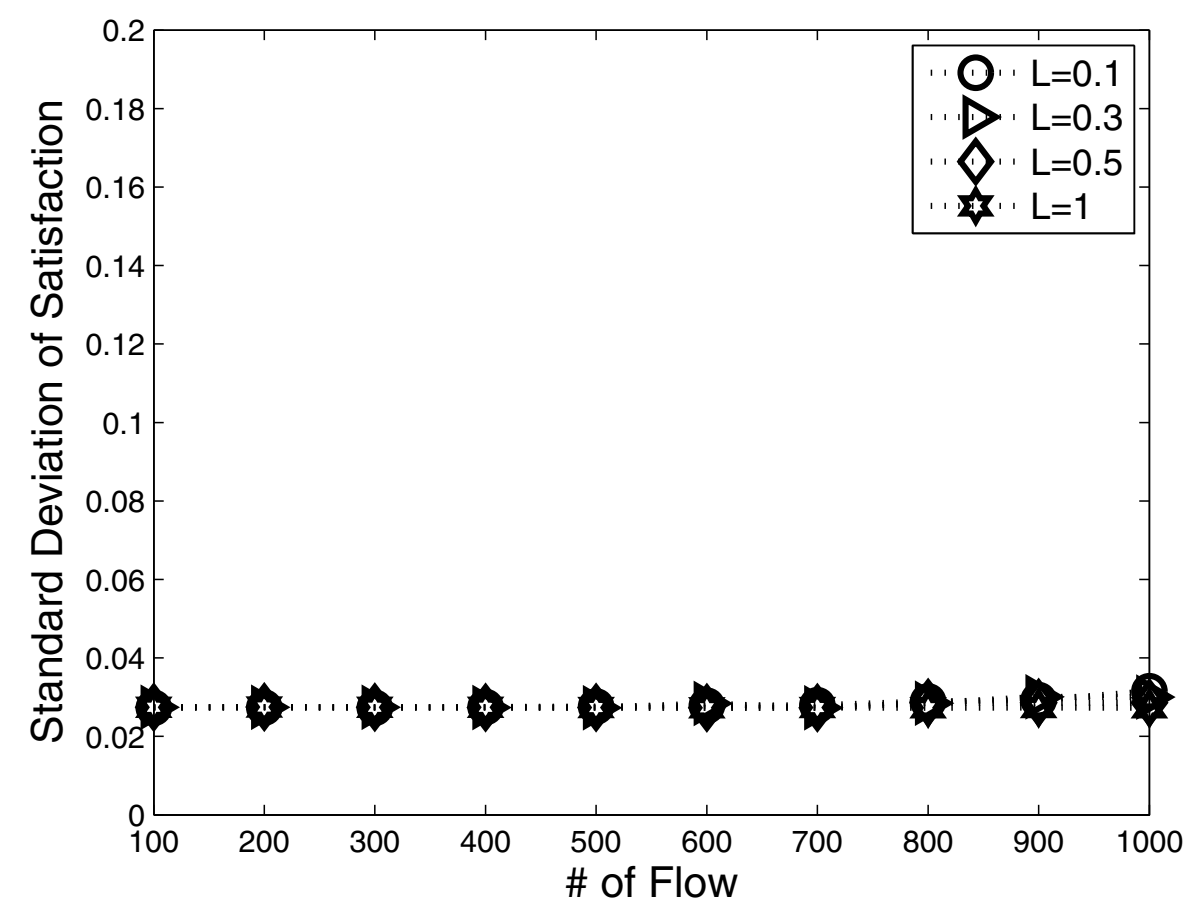

Figure 30 Standard deviation of satisfaction in MD_AwayFromBS with $S \_T H=0.9$.

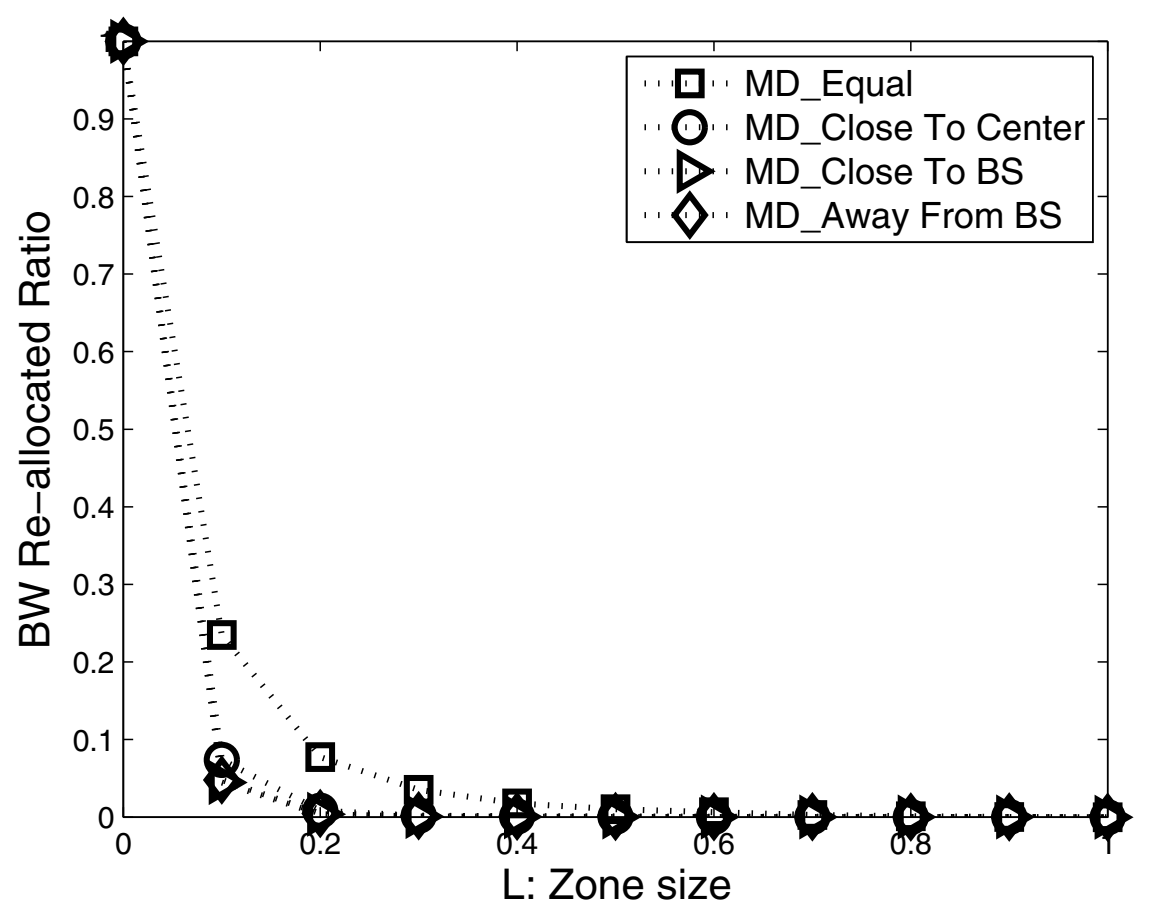

Figure 31 Bandwidth re-allocation ratio in flow $=100$ (light load). 


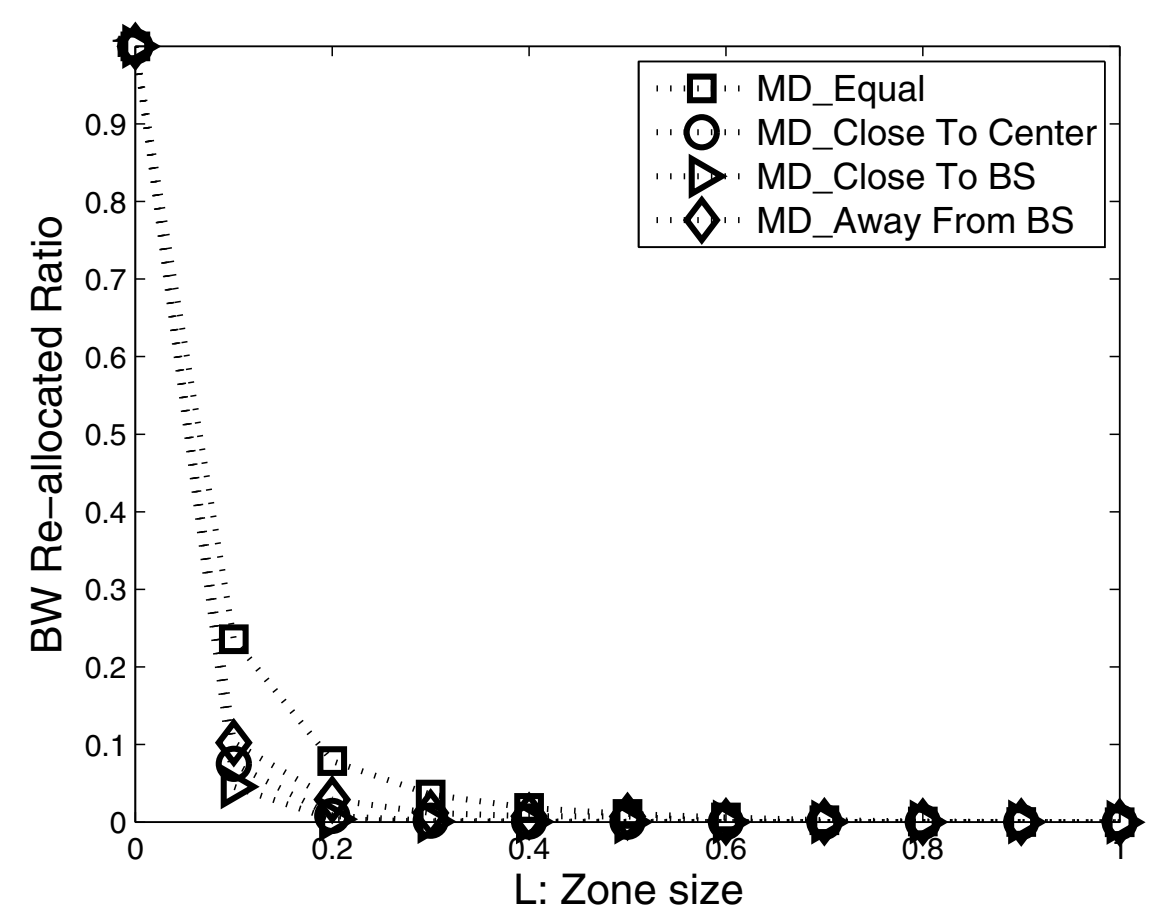

Figure 32 Bandwidth re-allocation ratio in flow $=500$ (middle load)

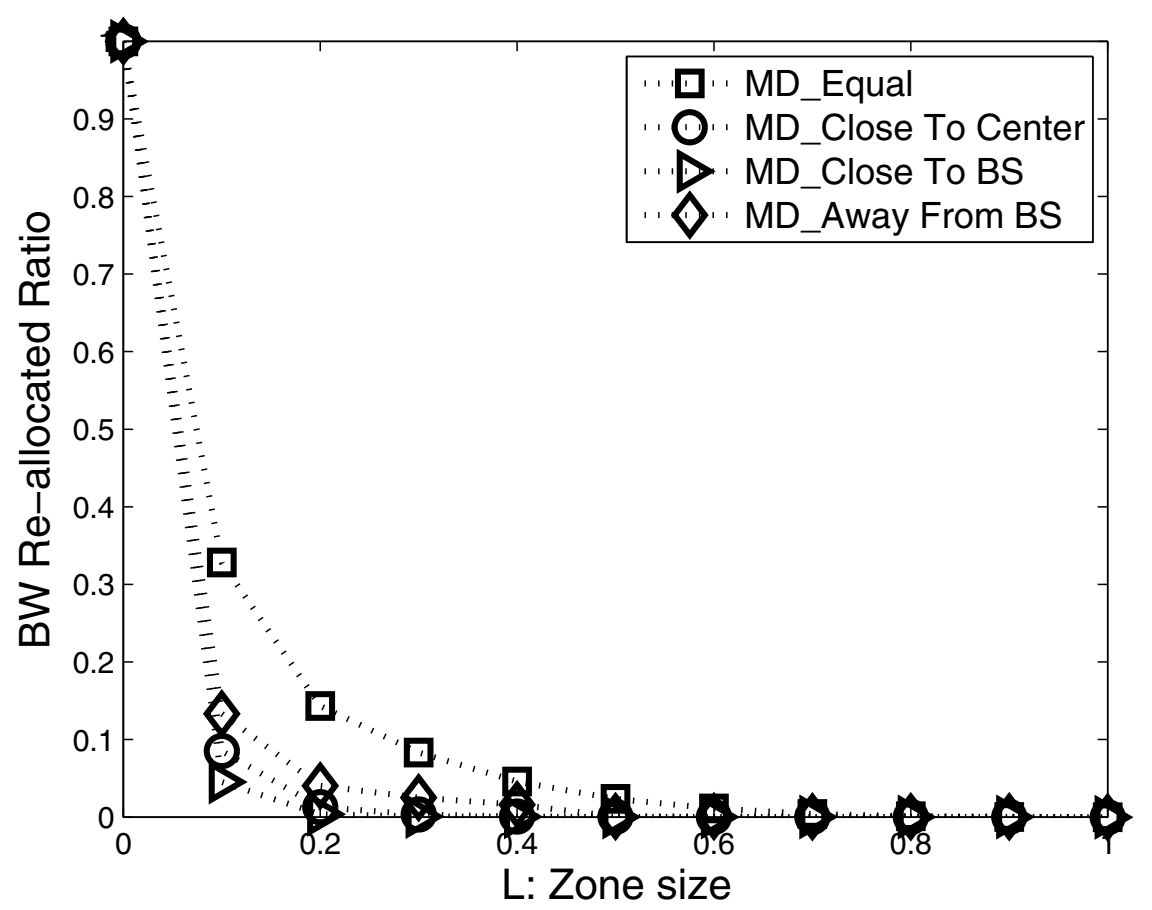

Figure 33 Bandwidth re-allocation ratio in flow $=1000$ (heavy load). 


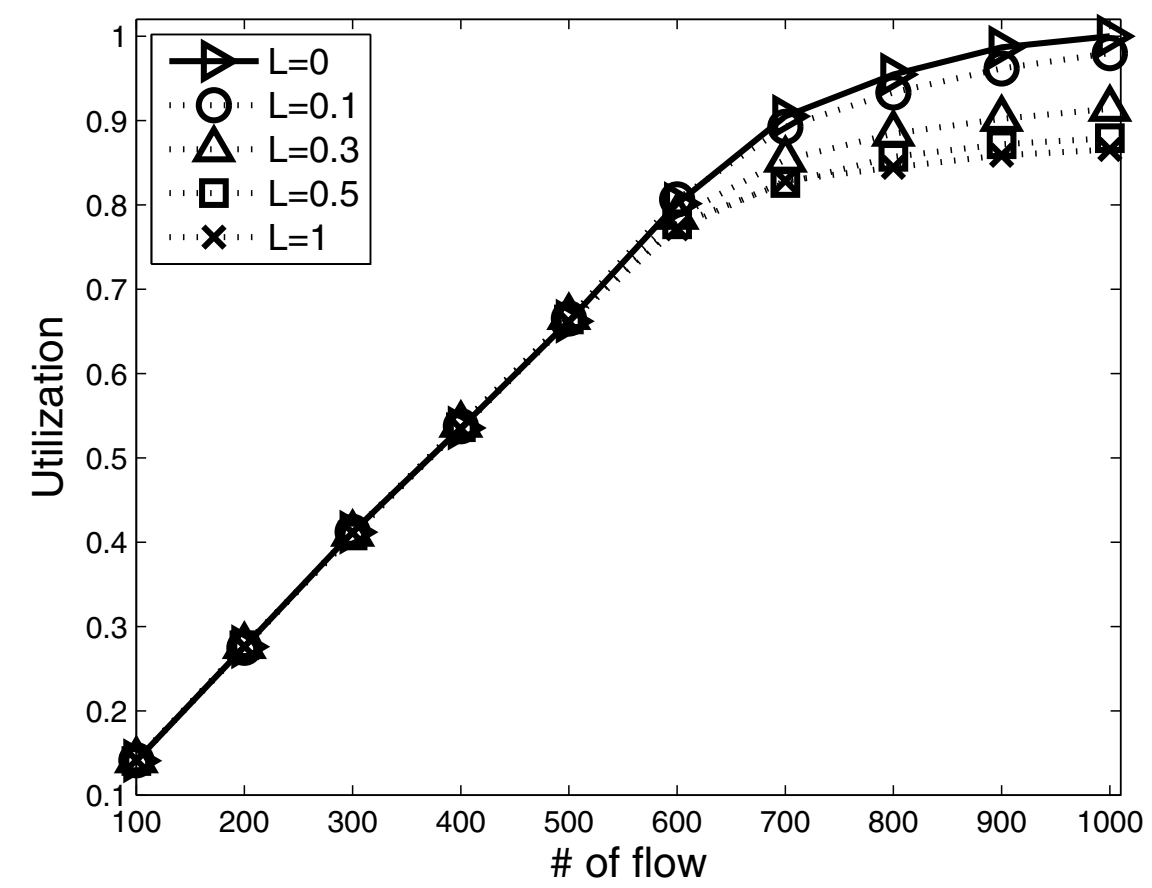

Figure 34 Bandwidth utilization in $S_{-} T H=0.9$.

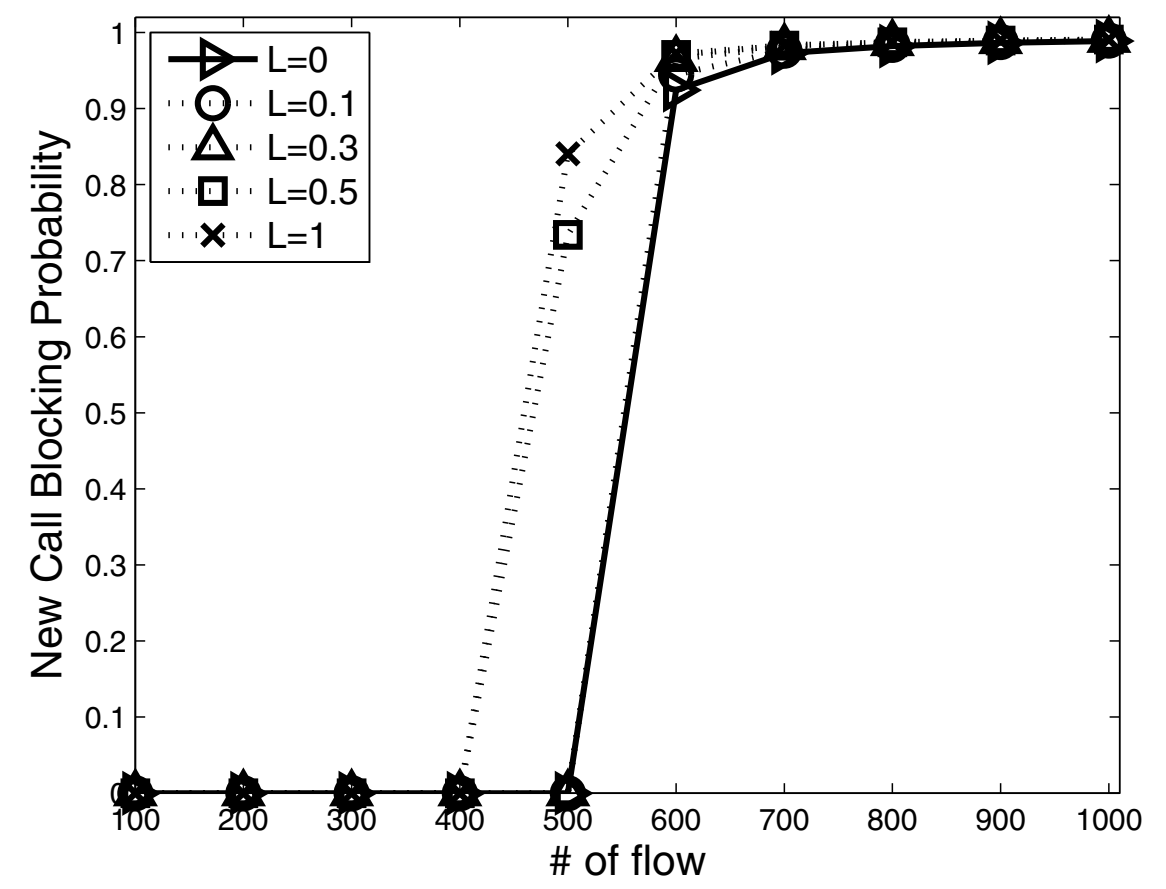

Figure 35 New call blocking probability in $S_{-} T H=0.9$. 
\# of flow higher 400, one is growing suddenly, the other one is growing gradually in Figures 36 to 39. The effect of zone size $L$ is obvious in the Handoff Call Degradation Ratio, which is reducing as the zone size increases. However, the $L=1$ has special characteristic and it shows the degradation ratio $=0$. When $L=1$, there is no chance for $\mathrm{MH}$ to occur inter-zone handoff.

In summary, a larger zone can reduce Handoff Call Degradation Ratio and Bandwidth Re-allocation Ratio, at the cost of more Bandwidth Allocation and New Call Blocking Probability.

\section{Conclusion}

IEEE 802.16 (WiMAX) Wireless Network technology is a popular research issue in recent years. It provides wider coverage of radio, faster wireless access, and the Quality-of-Service plays an important role in the standard for promoting the technology. For mobile multihop wireless network, IEEE 802.16j/MR network not only can supply large area wireless deployment, but also provide high quality network service to mobile users. In this paper, a novel Zone-based bandwidth management scheme is proposed to maintain mobile users in the IEEE 802.16-MR network. Based on our proposed scheme, mobile users can achieve their QoS satisfaction and requirement in the IEEE 802.16-MR network. In the topology analysis, the chessboard network topology is more suitable for bandwidth allocation. Simulation study has demonstrated our proposed scheme can meet user's requirement even mobile users have different mobility behavior. The larger zone size can effectively reduce QoS degradation and bandwidth re-allocation but decreases bandwidth utilization. It follows that Zone-based bandwidth management scheme can be strengthened and better ways of QoS supporting are needed for MHs in IEEE 802.16-MR network. Future work of the research is to design an adaptive Zone size scheme to select the appropriate zone size for mobile users with different movement and mobility distribution characteristic.

List of Abbreviations

BS: Base Station; BWA: Broadband Wireless Access; MAC: medium access control; MH: mobile host; MMR: mobile multi-hop relay; RS: Relay Station; WLAN: wireless LAN.

\section{Acknowledgements}

This work was supported in part by the National Science Council, Taiwan, R. O.C., under grant NSC 99-2221-E-164-006.

\section{Author details}

'Department of Information and Networking Technology, Hsiuping Institute of Technology, Taiwan, Republic of China ${ }^{2}$ Department of Computer Science and Information Engineering, National Chi Nan University, Taiwan, Republic of China

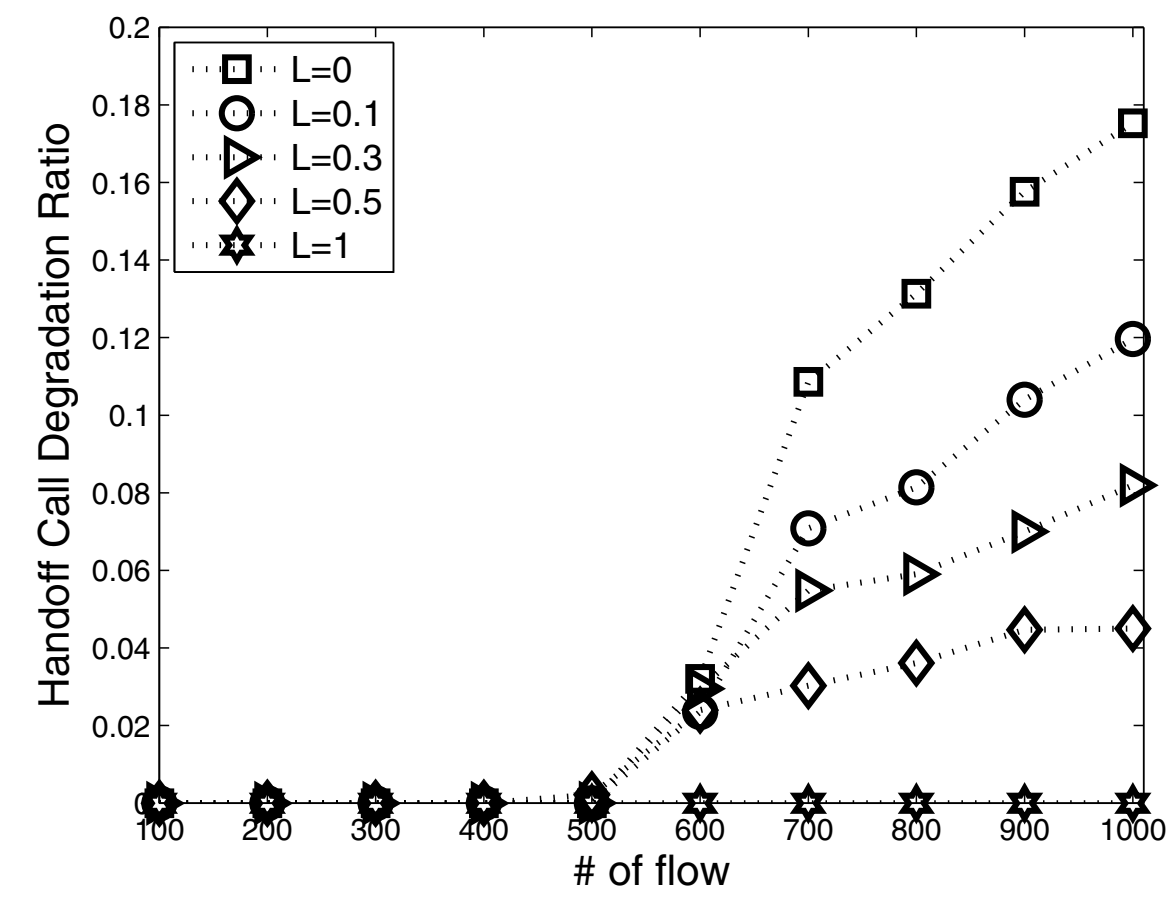

Figure 36 Handoff call degradation ratio in $S \_T H=0.9$. 


\section{Competing interests}

The authors declare that they have no competing interests.

\section{Received: 29 January 2011 Accepted: 16 June 2011}

\section{Published: 16 June 2011}

\section{References}

1. IEEE Std. 802.11, Wireless LAN medium access control (MAC) and physical layer (PHY) specifications, June 2003.

2. IEEE Std. 802.11e, Wireless LAN medium access control (MAC) and physical layer (PHY) specifications amendment 8: medium access control (MAC) quality of service enhancements, Nov. 2005.

3. 3GPP, http://www.3gpp.org/ M. Garcia-Martin, "3rd-Generation Partnership Project (3GPP) Release 5 requirements on the Session Initiation Protocol (SIP)," IETF Internet-Draft draft-ietf-sipping-3gpp-r5requirements-00.txt, Oct. 2002.

4. S Parkvall, E Englund, M Lundevall, J Torsner, Evolving 3G mobile systems: Broadband and broadcast services in WCDMA. IEEE Commun. Mag. 44(2), 68-74 (2006)

5. 3GPP TS 25.308, UTRA High Speed Downlink Packet Access (HSDPA); Overall description; Stage 2, vol. 5.7.0, Dec. 2004, http://www.3gpp.org/ftp/specs/ htmlinfo/25308.htm

6. IEEE Std. 802.16-2004, IEEE standard for local and metropolitan area networks-part 16: air interface for fixed broadband wireless access systems, Oct. 2004.

7. IEEE Std. 802.16e-2006, IEEE standard for local and metropolitan area networks-part 16: air interface for fixed broadband wireless access systemsamendment: physical and medium access control layers for combined fixed and mobile operation in licensed bands. Feb. 2006.

8. IEEE Std. 802.16-2009, IEEE standard for local and metropolitan area networks part 16: air interface for broadband wireless access systems. May 2009.

9. IEEE Std 802.16j-2009, IEEE standard for local and metropolitan area networks part 16: air interface for broadband wireless access systems amendment 1: multiple relay specification. June 2009.

10. H Wang, B He, DP Agrawal, Admission control and bandwidth allocation above packet level for IEEE 802.16 Wireless MAN. in Proceedings of 12th International Conference on Parallel and Distributed Systems (ICPADS 2006), vol. 1, July 2006, pp. 1-6

11. L Wang, F Liu, Y Ji, N Ruangchaijatupon, Admission control for nonpreprovisioned service flow in wireless metropolitan area networks. in Proceedings of 4th European Conference on Universal Multiservice Networks (ECUMN 2007), Feb. 2007, pp. 243-249

12. A Sayenko, O Alanen, J Karhula, T Hamalainen, Ensuring the QoS requirements in 802.16 scheduling. in Proceedings of 9th ACM International Symposium on Modeling Analysis and Simulation of Wireless and Mobile Systems (MSWiM 2006), Oct. 2006. pp. 108-117

13. X Bai, A Shami, KA Meerja, C Assi, New distributed QoS control scheme for IEEE 802.16 wireless access networks. in Proceedings of IEEE Global Telecommunications Conference (GLOBECOM 2006), Nov. 2006, pp. 1-5

14. J Chen, W Jiao, Q Guo, An providing integrated QoS control for IEEE 802.16 broadband wireless access systems. in Proceedings of IEEE 62nd Vehicular Technology Conference (VTC 2005Fall), vol.5, Sep. 2005, pp. 1254-1258

15. YW Chen, IH Peng, ST Guan, Dynamic bandwidth management for handoffs with RSVP in 802.16/WLAN environment. in Proceedings of Advanced Information Networking and Applications Workshops, 2007 (AINAW 07), vol. 2, May 2007, pp. 243-248

16. HY Wei, S Granguly, R Izmailov, ZJ Haas, Interference aware IEEE 802.16 WiMax mesh networks. in Proceedings of IEEE 61st Vehicular Technology Conference (VTC 2005Spring), vol. 5, May 2005, pp. 3102-3106

17. B Han, FP Tso, L Ling, W Jia, Performance evaluation of scheduling in IEEE 802.16 based wireless mesh networks. in Proceedings of IEEE International Conference on Mobile Adhoc and Sensor Systems (MASS 2006), Oct. 2006, pp. 789-794

18. B Han, W Jia, L Lin, Performance evaluation of scheduling in IEEE 802.16 based wireless mesh networks. J. Comput. Commun. 30(4), 782-792 (2007)

19. MS Kuran, G Gur, T Tuğcu, F Alagöz, Crosslayer routing scheduling in IEEE 802.16 mesh networks. in Proceedings of 1st International Conference on MOBILe Wireless MiddleWARE, Operating Systems, and Applications (MOBILWARE'O8), vol. 278, Feb. 2008, pp. 4-7
20. H Shetiya, V Sharma, algorithms for routing and centralized scheduling to provide QoS in IEEE 802.16 mesh networks. in Proceedings of 1st ACM Workshop on Wireless Multimedia Networking and Performance Modeling (WMuNeP 2005), Oct. 2005, pp. 140-149

21. S Xergias, N Passas, Ak Salkintzis, Centralized resource allocation for multimedia traffic in IEEE 802.16 mesh networks. Proc. IEEE. 96(1), 54-63 (2008)

22. Y Zhang, H Hu, HH Chen, QoS differentiation for IEEE 802.16 WiMAX mesh networking. Mobile Netw. Appl. 13(12), 19-37 (2008)

23. J Zhang, S Feng, W Ye, H Zhuang, MAC performance evaluation of IEEE 802.16j. in Proceedings of IEEE International Symposium on Informormational Science Engineering (ISISE), vol. 1, Dec. 2008, pp. 421-425

24. D Niyato, E Hossain, DI Kim, Z Han, Relaycentric radio resource management and network planning in IEEE 802.16j mobile multi-hop relay networks. IEEE Trans. Wireless Commun. 8(12), 6115-6125 (2009)

25. SR Yang, CC Kao, WC Kan, TC Shih, Handoff minimization through a relay station grouping algorithm with efficient radioresource scheduling policies for IEEE 802.16j multi-hop relay networks. IEEE Trans. Vehicul. Technol. 59(5), 2185-2197 (2010)

26. B Wang, M Mutka, Path selection for mobile stations in IEEE 802.16 multihop relay networks. in Proceedings of International Symposium on a World of Wireless, Mobile and Multimedia Networks (WoWMoM), June 2008, pp. 1-8

27. S Ann, KG Lee, HS Kim, A path selection method in IEEE 802.16j mobile multi-hop relay networks. in Proceedings of IEEE International Conference on Sensor Technologies and Applications (SENSORCOMM'08), Aug. 2008, pp. 808-812

28. L Erwu, W Dongyao, L Jimin, S Gang, J Shan, Performance evaluation of bandwidth allocation in $802.16 \mathrm{j}$ mobile multi-hop relay networks. in Proceedings of IEEE 65th Vehicular Technology Conference (VTC2007Spring), April 2007, pp. 939-943

29. AF Bayan, TC Wan, A scalable QoS scheduling architecture for WiMAX multi-hop relay network. in Proceedings of 2nd International Conference on Education Technology and Computer (ICETC), vol. 5, June 2010, pp. 326-331

30. YT Mai, CC Yang, YH Lin, Design of the crosslayer QoS framework for the IEEE 802.16 PMP networks. IEICE Trans. Commun. E91B(5), 1360-1369 (2008)

31. CC Yang, YT Mai, LC Tsai, Design of the QoS framework for the IEEE 802.16 mesh networks. Int. J. Commun. Syst. 22(12), 1543-1562 (2009)

32. PS Mogre, M Hollick, R Steinmetz, V Dadia, S Sengupta, Distributed bandwidth reservation strategies to support efficient bandwidth utilization and QoS on a per-link basis in IEEE 802.16 mesh networks. in Proceedings of IEEE 34th Conference on Local Computer Networks (LCN 2009), Oct. 2009, pp. 301-304

33. A Kapoor, VJ Ribeiro, An endtoend QoS aware greedy distributed scheduling framework for WiMAX mesh networks. in Proceedings of Second International Conference on Communication Systems and Networks (COMSNETS 2010, Jan. 2010), pp. 1-8

34. AK Talukdar, BR Badrinath, A Acharya, MRSVP: a resource reservation protocol for an integrated services network with mobile hosts. Wireless Netw. 7(1), 5-19 (2001)

35. CC Tseng, GC Lee, RS Liu, TP Wang, HMRSVP: a hierarchical mobile RSVP protocol. Wireless Netw. 9(2), 95-102 (2003)

36. E Fogelstroem, A Jonsson, C Perkins, Mobile IPv4 regional registration. IETF RFC 4857, June 2007

37. JY Chen, CC Yang, LS Yu, HH-MIP: an enhancement of mobile IP by home agent handover. EURASIP J. Wireless Commun. Netw. 2010 Article ID 653838, 14 (2010)

doi:10.1186/1687-1499-2011-15

Cite this article as: Mai and Chen: Design of zone-based bandwidth management scheme in IEEE 802.16 multi-hop relay networks. EURASIP Journal on Wireless Communications and Networking 2011 2011:15. 